\title{
On the state of deformation in a polycrystalline material in three-dimension: elastic strains, lattice rotations, and deformation mechanisms
}

\author{
${ }^{1,3}$ Hamidreza Abdolvand, ${ }^{2}$ Jonathan P Wright, ${ }^{3}$ Angus J Wilkinson
}

\author{
${ }^{I}$ Department of Mechanical and Materials Engineering, Western University, Spencer Engineering \\ Building, London, Ontario, Canada, N6A $5 B 9$ \\ ${ }^{2}$ ESRF, 71 Avenue Des Martyrs, 38000 Grenoble, France
}

${ }^{3}$ Department of Materials, University of Oxford, Parks Road, Oxford, UK, OXI $3 \mathrm{PH}$

\begin{abstract}
The deformation of polycrystalline zirconium is analysed at the individual grain level using threedimensional Synchrotron X-ray Diffraction (3D-XRD) in combination with a Crystal Plasticity Finite Element (CPFE) model. The effects of elastic strains and lattice rotations on the shape and position of diffracted peaks are studied in detail. For this purpose, the three dimensional measured microstructure of a commercially pure zirconium (CPZr) sample was imported into a crystal plasticity finite element (CPFE) model to simulate the deformation of measured grains. The calculated lattice rotations and strains of the selected most active grains were subsequently used to forward simulate diffracted peaks. The simulated peaks are then compared to the 3D-XRD measured ones. It is shown that although the sample was deformed to $1.2 \%$ strain, soft grains exhibit significant peak smearing. Peak broadening is mostly affected by the deformation induced lattice rotation and less affected by the elastic strains. Further, the comparison of CPFE modelling and 3D-XRD results confirms that prism and basal slip are the most active systems and pyramidal $\langle\mathrm{c}+\mathrm{a}\rangle$ is present but only slightly active. A method is developed to determine the activity of possible slip systems. As an example, by comparing the simulated and measured peak positions as well as the rotations of the most affected diffraction peaks, it is shown that pyramidal $<a>$ slip system is not active significantly at room temperature.
\end{abstract}

Keywords: deformation mechanism, three dimensional synchrotron diffraction, crystal plasticity, finite element, hexagonal close packed, polycrystals

\section{Introduction}

The improvement of materials constitutive equations demands continued development of new experimental techniques which can provide the information required for validating or updating the models. From the materials deformation prospective, these experiments can be conducted at different length scales provided that the necessary data required for predicting material behaviour can be extracted. For instance, at nano and micro scales, cantilever beams or pillars can be used to characterize mechanical properties of engineering materials. These experiments are mainly conducted by milling micro-beams which will be deflected while the force-displacement curve is recorded [1-6]. The measured force-displacement curve and the orientation of the pillar can then be compared to a crystal plasticity or dislocation dynamic simulation to extract critical resolved shear stress (CRSS) values of each individual active system. For instance, by milling cantilever beams with particular orientations, Gong and Wilkinson have isolated each active slip system in CPZr micro-cantilevers and measured CRSS values accounting for size effects and used these CRSS values to predict bulk polycrstal deformation using CPFE [7]. Further, in a set of carefully designed experiments, the response of $\alpha$ and $\beta$ titanium pillars to various applied strain rates and the interaction between the two phases of the composite pillar were studied by Zhang et al $[8,9]$ and Jun et al $[10,11]$. 
A more advanced version of these experiments is when micro-Laue diffraction is used to record the variation of lattice parameters of the single crystal which can consequently be transformed into elastic strain and stress, i.e. the evolution of state of deformation in a single crystal can be studied [12-15]. For instance, Kirchlechner et al [16] studied in-situ compression of copper micro-pillars in which it was shown that instrumental constraint led to dislocation storage at the top and bottom of the sample. More recently, they conducted in-situ fatigue experiments on copper in which the evolution of diffraction peaks as a function of number of fatigue cycles were studied [17]. Further, in-situ deformation of $\mathrm{Ni}$ micro-pillars with micro-Laue diffraction was conducted by Maa $\beta$ and Uchic [18] where the size dependent strain hardening and the exhaustion of hardening was studied. While all of these experiments provide invaluable insight toward understanding deformation mechanism of crystals, they all suffer from the possible damage that can be induced during $\mathrm{Ga}^{+}$milling of the micro-beam [19] and the socalled "size effect" problem.

An alternative experimental approach is to study deformation of individual grains or cluster of grains in the bulk of the investigated polycrystal. This is feasible thorough the use of neutron or synchrotron $\mathrm{X}$-ray diffraction techniques. One of the methods that has been used predominantly is to study peak broadening resulting from strain or orientation heterogeneity within the grain(s) of the polycrystal [2025]. In conventional powder diffraction techniques, an area detector is used to record the diffracted patterns. For a perfect single crystal, the monochromatic diffracted beam will leave a single spot on the "perfect" detector. However, the stress field associated with dislocations, if they are present, will distort the perfect lattice which results in perturbation of the diffracted beam and peak broadening. This can be used to study deformation mechanisms; for instance, through the interpretation of peak broadening, Guilionda et al [26] used high energy X-ray diffraction to measure the evolution of dislocation densities in a deformed Al-Mg alloy. Similarly, Wang et al [27] used X-ray diffraction to characterize microstructural evolution of a ferritic-martensitic steel undergoing tensile load at room and higher temperature. In this study, through the analyses of peak broadening, the authors could separate the two stages of dislocation multiplication and formation of dislocation walls. More recently, this technique was successfully used to study deformation mechanisms of neutron irradiated nuclear materials $[28,29]$.

Analysis of diffraction peak broadening measured by three-dimensional X-ray diffraction technique (3D-XRD) has become the focus of recent studies [30-33]. Depending on the experimental set-up, more than 100 peaks can be assigned to each probed grain of a polycrystal. If grains are annealed or recrystallized, circular diffraction peaks will appear on the detector, while plastic deformation will induce orientation distribution within grains and consequently lead to peak smearing. This is a unique advantage because the state of stress within grains and their associated neighbouring environments can be measured and directly linked to changes in dislocation content which affect the peak shape, making it feasible to explore deformation mechanism of the polycrystal more accurately. For instance, Jakobsen et al [34], used 3D-XRD to study state of deformation in copper and analyse the structure of dislocation at a fixed external tensile strain. This technique was further developed to study deformation of copper under cyclic loading [35]. It was used to show that highest Schmid factor slip systems in a ferritic steel are responsible for orientation spread within the investigated grains in an experiment conducted by Oddershede et al [36].

In this paper, the effects of strain and orientation spread on the peak smearing of the grains of a CPZr sample are studied in detail. The CPZr sample has Hexagonal Close-Pack (HCP) crystal structure with high elastic and plastic anisotropy which make it suitable to study possible active slip systems. After post-processing the measured 3D-XRD data, the initial "undeformed" microstructure was imported into a CPFE model to simulate deformation of the sample. Grains for which deformation was predominately on a selected slip system in the simulated polycrystal were then selected for further analysis. The calculated orientation and strain spread of these selected grains were imported into a code that forward simulates diffraction patterns. As grain, and grain neighbour, shapes and orientations are the matched across the experiment and simulations comparison of simulated and measured diffraction patterns allow 
assessment of the dominant deformation mechanism of the selected grains. A particular focus was in using this new methodology to assess the extent of any pyramidal $<\mathrm{a}>$ slip, but grains with deformation predominately on prism $<\mathrm{a}>$, basal $<\mathrm{a}>$ and pyramidal $<\mathrm{c}+\mathrm{a}>$ were also critically examined. The pyramidal $<\mathrm{a}>$ slip system has been suggested as active in some circumstance in $\mathrm{Zr}$ alloys, for example at higher temperatures [6], and for some textures [Liu et al, Mat Sci Eng A. 719, (2018), 147-154], while modelling works have often included pyramidal $<a>$ slip even at room temperature and found reasonable comparison to macroscopic behaviour [eg Lebensohn, Sanchez \& Pochettino, Scripta Metall., 30 (1994), 481-486, Philippe et al, Acta Metall. Mater., 43 (1995), 1619-1635, Tome', Lebensohn \& Kocks, Acta Metall. Mater. 39, (1991) 2667, Xu, Holt \& Daymond, J. Nucl. Mater., 373 (2008), 217-225]. There are however other studies concluding that at room temperature in $\mathrm{Zr}$ alloys pyramidal $<\mathrm{a}>$ slip systems are not active $[4,5]$. Similar ambiguities exist in the literature for the related Ti alloys. The proposed new methodology has the advantage of being able to select from a large sample volume some particular grains where it is most likely that pyramidal $<\mathrm{a}>$ slip might occur. This should increase the sensitivity and reduce the ambiguity of the assessment compared to simply using the macroscopic response.

\section{Simulating diffraction patterns}

A dog-bone CPZr sample was deformed in-situ to study deformation of individual grains [37]. The sample was machined from a CPZr bar that was annealed and recrystallized, with the loading axis aligned with axis of the bar (see figure 1). After polishing it was revealed that the sample cross section was $0.5 \times 0.53 \mathrm{~mm}^{2}$. The sample was initially mounted onto a deformation rig which was fixed onto a rotating stage. Synchrotron X-ray beam with the energy of $78.39 \mathrm{keV}$ and the vertical size of 25 microns was shone on to the sample which was positioned $374.5 \mathrm{~mm}$ away from a 2D detector (see Fig. 1a). The sample and the tensile stage were then rotated about the loading axis so that the crystallographic planes of different grains can come into diffraction condition. The stage was initially rotated from $\omega=$ $-234.5^{\circ}$ to $-125.5^{\circ}$ and then from $-54.5^{\circ}$ to $54.5^{\circ}$ with the step size of $\Delta \omega=0.25^{\circ}$, and a collection time of $0.25 \mathrm{~s}$ per pattern. An example of the measured patterns are shown in Fig. 1b. Measurements were performed at four deformation steps, i.e., preload, near the onset of plasticity where applied strain was $0.6 \%$, applied strain of $1.2 \%$, and unload. At each measurement step, 15 layers of the sample were probed by the X-ray beam which led to measuring more than 11,000 grains. The post-processing of the diffraction patterns, including peak search, indexing peaks, and determining grains, was mainly done by the use of ImageD11 and the subroutines embedded into Fable (https://sourceforge.net/p/fable/wiki/Home/). The peak search was performed on the first 12 complete rings, while the incomplete rings close to the corners of the detector were cut-off. Detailed process of indexing patterns are discussed elsewhere [38]. For each grain it was possible to measure relative volume, centre-of-mass position (COM), average crystallographic orientation, elastic strain, and stress. On average more than 100 peaks were identified an assigned to each measured grain. At this stage it was possible to correlate grains from each loading step using their measured COM, orientation, and neighbours. After correcting the possible rigid body movement, it was possible to track more than 5500 grains across all of the loading steps.

In order to understand the effects of active slip systems on the shape of the diffraction peaks, it is necessary to understand the geometry of the test set-up and the effects of the key parameters on the appearance of a peak. The details of the 3D-XRD test geometry and the possible outcome of each test set-up is explained in the reference [39]; here, we will focus on the "far-field" measurement. This setup is shown in Fig. 1a where the distance between the center of the sample to the detector is $D$, the angle between the diffracted $\left(\vec{R}_{1}\right)$ and incoming $\left(\vec{R}_{0}\right)$ beams is $2 \theta$, and the angle between the vertical line on the detector and a given peak is $\eta$ with clockwise rotation about the X-ray beam direction. The condition for a crystallographic plane to diffract is: 


$$
\vec{q}=\frac{\vec{R}_{0}-\vec{R}_{1}}{\lambda}=\vec{r}_{h k l}^{g}
$$

Where $\vec{q}$ is the scattering vector that bisects the angle between $\vec{R}_{1}$ and $\vec{R}_{0}, \lambda$ is the X-ray wavelength, and $\vec{r}_{h k l}^{g}$ is the reciprocal lattice vector that is normal to the ( $\left.h k l\right)$ plane. The superscript $g$ indicates that the lattice vector is given in the "global" coordinate system.

Three coordinate systems are used to extract the equations. The "global" coordinate system $(g)$ is fixed and has the $\mathrm{x}$, and $\mathrm{y}$ directions coinciding with the X-ray beam, and transverse directions, respectively. The global z-axis is perpendicular to the X-ray beam direction and points to the north. When the sample is perfectly aligned, the global z-axis coincides with the loading direction. The sample coordinate system $(s)$ is attached to the sample and rotates about the loading direction, i.e. the global z-axis. The sample coordinate system coincides with the global coordinate system at $\omega=0$. The last coordinate system is the crystal coordinate system (c) which is attached to the HCP crystal. Hence, any lattice vector given in the crystal coordinate system $\left(\vec{r}_{h k l}^{c}\right)$ can be transform to the one in the sample coordinate system $\vec{r}_{h k l}^{s}$ using Eq. 2:

$$
\vec{r}_{h k l}^{S}=R_{c}^{S} \vec{r}_{h k l}^{c}
$$

where $R_{c}^{S}$ is the rotation tensor that transforms crystal coordinate system to the sample coordinate system. Since the sample coordinate system rotate about the global z-axis, $\vec{r}_{h k l}^{S}$ can be presented in the global coordinate system using Eq. 3:

$$
\vec{r}_{h k l}^{g}=R^{\omega} \vec{r}_{h k l}^{s} \text { where } R^{\omega}=\left[\begin{array}{ccc}
\cos \omega & \sin \omega & 0 \\
-\sin \omega & \cos \omega & 0 \\
0 & 0 & 1
\end{array}\right]
$$

With the geometry shown in Fig. 1a, the unit vector of $\vec{R}_{1}$ is:

$$
\vec{R}_{1}=\left[\begin{array}{c}
\cos 2 \theta \\
-\sin 2 \theta \sin \eta \\
\sin 2 \theta \cos \eta
\end{array}\right]
$$

By substituting Eq. 4 into Eq. 1 and knowing that the unit vector of $\vec{R}_{0}$ is $[1 ; 0 ; 0]$, the scattering vector can be represented as:

$$
\vec{q}=\left[\begin{array}{c}
-\sin \theta \\
-\cos \theta \sin \eta \\
\cos \theta \cos \eta
\end{array}\right]
$$

The crystallographic orientation of a grain is normally given in the form of Euler angles $\left(\varphi_{1}, \phi, \varphi_{2}\right)$ which can be used to calculate $R_{c}^{S}$ and to convert any vector in the crystal coordinate system to the sample coordinate system using Eq. 2. By substituting Eq. 5 into Eq. 1 and using Eq. 2 and 3, for a given $\vec{r}_{h k l}^{S}$ we have

$$
\left[\begin{array}{ccc}
\cos \omega & \sin \omega & 0 \\
-\sin \omega & \cos \omega & 0 \\
0 & 0 & 1
\end{array}\right]\left[\begin{array}{l}
r_{x}^{s} \\
r_{y}^{s} \\
r_{z}^{s}
\end{array}\right]=\left[\begin{array}{c}
-\sin \theta \\
-\cos \theta \sin \eta \\
\cos \theta \cos \eta
\end{array}\right]
$$

where $r_{x}^{S}, r_{y}^{S}$, and $r_{z}^{S}$ are the components of $\vec{r}_{h k l}^{S}$.

Since the energy of the incident X-ray beam is known $(78.39 \mathrm{keV})$, the angle $\theta$ for a given $(h \mathrm{kl})$ plane can be calculated using the Bragg's Law. Hence, the $\omega$ at which the $(h k l)$ plane fulfils the diffraction condition can be calculated by solving the following equation: 


$$
r_{x}^{S} \cos \omega+r_{y}^{S} \sin \omega=-\sin \theta
$$

The solution to the Eq. 7 provides the $\omega$ that can be subsequently substituted into Eq. 6 to calculate $\eta$. Hence, for a given grain orientation and X-ray beam energy, several sets of $(\theta, \eta, \omega)$ can be calculated for a given $(h k l)$ plane.

The position of a peak on the detector can be determined by intersecting the line that passes through the centre of the mass of a grain (COM), at a given $\omega$, with the direction of $\vec{R}_{1}$ and the detector plane. Hence, for a given $(h k l)$ plane, the position of the diffracted peak on the detector can be calculated using Eq. 8.

$$
\begin{aligned}
& P_{y}=\frac{R_{1 y}}{R_{1 x}} D-\frac{R_{1 y}}{R_{1 x}} C O M_{x}^{\omega}+\operatorname{COM}_{y}^{\omega} \\
& P_{z}=\frac{R_{1 z}}{R_{1 x}} D-\frac{R_{1 z}}{R_{1 x}} C O M_{x}^{\omega}+\operatorname{COM}_{z}^{\omega}
\end{aligned}
$$

where $R_{1 x}, R_{1 y}$, and $R_{1 z}$ are the components of $\vec{R}_{1}$, and $C O M_{i}^{\omega}$ represents the $\mathrm{i}^{\text {th }}$ component of the COM at the diffracting $\omega$.

If the Euler angles for each grain is known, it is possible to calculate the rotation matrix $R$ that transforms any vector in the crystal coordinate to the global coordinate [40]. For the sample that is perfectly aligned, the Euler angles (or $R$ ) correlate with $\theta, \eta$, and $\omega$ through the following equation:

$$
R=R_{c}^{S} R^{\omega} \text { or } R \vec{r}_{h k l}^{c}=\vec{q}_{\theta, \eta, \omega}
$$

For simulating diffraction peaks we assume that: (a) the X-ray beam is absolutely monochromatic, i.e. energy of the beam is constant across the beam, (b) the beam intensity is uniform, (c) grain size and shape will not affect the shape of the diffraction peaks, and (d) detector is perfect with no distortion. The assumption (a) is made based on the fact that the variation of X-ray energy or $\mathrm{dE} / \mathrm{E}$ is $2 \times 10^{-3}$ which corresponds to 0.008 degrees variation for the highest measured $\theta$. Given that the average grain size for the sample is about $30 \mu m$ (See suplimentary Fig S2), it is sensible to assume that the intensity of the incident beam is nearly uniform over the grain volume. Also, since the detector pixel size is $50 \mu \mathrm{m}$, the grain size and shape will have sub-pixel (order of 30/50) effects on the calculation of peak position (see Eq. 8).

Fig1. Here

\section{Crystal plasticity modelling}

A crystal plasticity user material (UMAT) subroutine for the Abaqus finite element (FE) solver was used for simulating deformation of each grain. A brief description of the key equations used in the subroutine is given here; comprehensive explanations are given elsewhere [41,42]. At the beginning of each time increment, Abaqus passes strain and time increment data into the UMAT in which the new

state of stress, solution dependent state variables, and the Jacobian matrix $\left(\frac{\partial \Delta \sigma}{\partial \Delta \varepsilon}\right)$ will be calculated and returned to the FE solver. The total strain increment $(\Delta \varepsilon)$ can be decomposed to the elastic part $\left(\Delta \varepsilon^{e l}\right)$ and the plastic $\left(\Delta \varepsilon^{p l}\right)$ part. For the CPZr sample used in this study, the c-axis of the HCP polycrystals are mostly in compression, and since the deformation is applied to small strains, the effects of twinning is neglected. This was confirmed by post EBSD analysis of the deformed sample where just a few twins were observed (see supplementary file). Hence, the plastic strain rate can be calculated from the slip rate $\left(\dot{\gamma}^{\alpha}\right)$ : 


$$
\begin{aligned}
\dot{\varepsilon}^{p l} & =\sum_{\alpha=1}^{N^{s p l}} P^{\alpha} \dot{\gamma}^{\alpha} \\
P^{\alpha} & =\operatorname{sym}\left(S^{\alpha}\right) \text { where } S^{\alpha}=d^{\alpha} \otimes n^{\alpha}
\end{aligned}
$$

In which $P^{\alpha}$ is the symmetric part of the Schmid tensor $\left(S^{\alpha}\right)$ for the slip system $\alpha, d^{\alpha}$ is the direction of the slip and $n^{\alpha}$ is the normal to the slip plane. Time integration of Eq. 9 will return the plastic strain increment. The slip rate of the slip system $\alpha$ can be calculated using Eq. 11 [43]:

$$
\dot{\gamma}^{\alpha}=\dot{\gamma}_{0}\left|\frac{\tau^{\alpha}}{g^{\alpha}}\right|^{n} \operatorname{sign}\left(\frac{\tau^{\alpha}}{g^{\alpha}}\right)
$$

where $\dot{\gamma}_{0}$ is a reference shear strain rate, $\tau^{\alpha}$ is the resolved shear stress acting on the slip system $\alpha$, and $g^{\alpha}$ is the current critical resolved shear stress (CRSS) of the slip system $\alpha$. At room temperature, plastic deformation of CPZr is mostly controlled by prism $\langle 11 \overline{2} 0\rangle$, basal $\langle 11 \overline{2} 0\rangle$, and pyramidal $\langle 11 \overline{2} 3\rangle$ slip systems. The CRSS values used in this study are $82 \mathrm{MPa}, 109 \mathrm{MPa}$, and $287 \mathrm{MPa}$, respectively, with no further hardening (ie $g^{\alpha}$ is independent of time). These CRSS values were established by scaling down the CRSS values reported by Gong et al [53] from micro-cantilever testing of the same batch of $\mathrm{CPZr}$ to allow for a change in strain rate and the stress relaxation occurring during the sample alignment for 3D-XRD measurement. Further, the values of the constants used in Eq. 10 for all of the simulations conducted in this paper are provided in the supplementary table 1 . With these parameters it was possible to capture both the distribution of stress for the modelled volume as well as that of individual grains. In Fig. 1c, the average measured stress-strain curve is compared to the calculated one. During the 3DXRD experiment, for each measurement step, the sample was firstly strained to $0.6 \%$ or $1.2 \%$ and then under strain-hold it was realigned and re-centred. This step was crucial for tracking individual grains across all of the loading-unloading steps. The realignment process took approximately 30 minutes during which most of the stress relaxation happened. Once the sample was realigned, diffraction patterns were collected. This step took about 7.5 hours over which less stress relaxation was observed. The measured macroscopic stresses at the maximum load, after alignment, and at the end of measurement are shown with black stars in Fig 1c. The weighted average of the stresses measured for all of the probed grains are shown with green triangle which coincide with the macroscopic stresses measured at the end of the each step. As such, the CRSS values of the CPFE model were selected so that the calculated average stress-strain curve coincide with those measured at the end of each loading step.

The shear stress acting on each slip system can be calculated from the Kirchhoff stress $(\Psi)$ through the following equation:

$$
\tau^{\alpha}=P^{\alpha}: \Psi
$$

The Jaumann rate of Kirchhoff stress $(\breve{\Psi})$ is related to the elastic part of the rate of deformation $\left(\dot{D}^{e l}\right)$ and the elastic stiffness tensor $(\mathbb{C})$ as:

$$
\breve{\Psi}=\mathbb{C}: \dot{D}^{e l} \text { where } \breve{\Psi}=\dot{\Psi}-\dot{\Omega}^{e l} \Psi+\Psi \dot{\Omega}^{e l}
$$

where $\dot{\Omega}^{e l}$ is the elastic part of the spin tensor. The deformation and the rotation rates are correlated to the symmetric and asymmetric parts of the velocity gradient $(L)$ as:

$$
\left(\dot{D}^{e l}+\dot{D}^{p l}\right)+\left(\dot{\Omega}^{e l}+\dot{\Omega}^{p l}\right)=\operatorname{sym}(L)+\operatorname{asym}(L)
$$

and the plastic part of the rotation increment is correlated to the plastic shear rate and asymmetric part of the Schmid tensor $\left(W^{\alpha}\right)$ :

$$
\dot{\Omega}^{p l}=\sum_{\alpha=1}^{N^{s p l}} W^{\alpha} \dot{\gamma}^{\alpha}
$$

The elastic modulus of the single crystal $\mathrm{CPZr}$ used in this study is the one determined by Fisher and Renken [44] : $\mathrm{C}_{11}=143.5 \mathrm{GPa}, \mathrm{C}_{33}=164.9 \mathrm{GPa}, \mathrm{C}_{12}=72.5 \mathrm{GPa}, \mathrm{C}_{13}=65.4, \mathrm{GPa}$, and $\mathrm{C}_{44}=32.1 \mathrm{GPa}$.

For forward simulation of diffraction spots in this paper, the updated Euler angles for each grain is used. At the beginning of simulation, each grain has a unique set of Euler angles that is measured by 3DXRD 
experiment. These Euler angle were assigned to the elements of the grain (see below) and were updated using the elastic part of the spin tensor:

$$
\begin{aligned}
& \dot{\varphi}_{1}=-\dot{\Omega}_{12}^{e l}-\dot{\varphi}_{2} \cos \phi \\
& \dot{\phi}=-\dot{\Omega}_{23}^{e l} \cos \varphi_{1}-\dot{\Omega}_{31}^{e l} \sin \varphi_{1} \\
& \dot{\varphi}_{2}=-\dot{\Omega}_{23}^{e l} \frac{\sin \varphi_{1}}{\sin \phi}+\dot{\Omega}_{31}^{e l} \frac{\cos \varphi_{1}}{\sin \phi}
\end{aligned}
$$

The measured COMs and relative volumes of grains were used to simulate grain shapes using a weighted Voronoi scheme and import the simulated microstructure into the Abaqus finite element solver. Since it is computationally costly to simulate heterogeneous deformation of all of the measured grains, only a subset of grains were imported into the FE solver. These grains are located in the centre of the probed volume and fall into a cube of $200 \mu \mathrm{m}$ side length for models S4. This simulation cube was then discretised with C3D8 elements, i.e., linear cube elements with 8 nodes and 8 integration points. Once the cube was meshed, it was possible to determine the position of each element in the cube. At this stage, this position was substituted into Eq. 17 for assigning a unique grain to the element:

$$
C_{i}=\left\{X \in R^{d} \mid\left\|X-s_{i}\right\|^{2}-w_{i}^{2}<\left\|X-s_{j}\right\|^{2}-w_{j}^{2}, i \neq j\right\}
$$

Where $\mathrm{X}$ is the position vector of the element $C_{i}$ in the cube, $s_{i}$ is the position vector of the seed point of the grain $i$, and $w_{i}$ is the radius of the same grain measured by 3DXRD. For determining the seed point of each grain, Eq. 17 is solved in two steps. In the first step, $s_{i}$ is replaced by the measured $\mathrm{COM}_{\mathrm{i}}$ of the grain $\mathrm{G}_{\mathrm{i}}$. This result in a new $\mathrm{COM}_{\mathrm{b}}$ for $\mathrm{G}_{\mathrm{i}}$ which does not necessarily coincide with the actual measured $\mathrm{COM}_{\mathrm{i}}$. In the second iteration, $s_{i}$ is set equal to $2 \mathrm{COM}_{\mathrm{b}}-\mathrm{COM}_{\mathrm{i}}$. It is shown that the second iteration provides a much better estimation of grain boundaries [45]. To examine the convergence of the simulations reported in this paper, the cube was meshed using different element sizes the results of which are provided in the supplementary information.

For simulating the experiment, each model was deformed at the same strain rate that was used in the experiment. In the first step, the model was cooled down from $600^{\circ} \mathrm{C}$ to account for the thermal residual stresses that can develop as a result of heat treatment. Periodic boundary conditions were applied on each of the surfaces of the simulation cube following the method described in [41]. A tensile elongation was applied along the vertical $\mathrm{z}$-axis at a rate matched to the experiment $\left(2.64 \times 10^{-5} \mathrm{~s}^{-1}\right)$, while in the transverse $\mathrm{x}$ and $\mathrm{y}$ axes the contraction was left free and the model allowed to relax to zero transverse net force. The thermal coefficients of expansion for CPZr single crystal are $(5.3,5.3,10.1) \times 10^{-6} 1 /{ }^{\circ} \mathrm{C}$ along the crystal (a, b, c) axis [46]. During the cooling step, this coefficient was multiplied by the temperature increment to calculate the thermal strain at each Integration Point (IP). Since the Euler angles and rotation matrix for each grain is given from the 3D-XRD experiment, the thermal strain is rotated into the global coordinate system. No mechanical load was applied to the model boundaries during the cooling step, and residual stresses develop as a result of local constraints imposed by the neighbouring grains.

\section{Linking CPFE to the simulation of diffraction peaks}

In a 3D-XRD experiment, the average stress, strain, orientation, COM, and volume of each grain in a polycrystal can be measured. Since many IPs were assigned to each grain in CPFE modelling, it was possible to calculate the variation of stress, strain and orientation resulting from grain-grain interaction. Two main input models were used for FE analysis; for the model S4, the grains that fall into a volume with the dimension of $200 \times 200 \times 200 \mu \mathrm{m}^{3}$ were used for simulating microstructure. This model is 
shown in Fig. 1d. The centre of the cube coincides with the centre of the volume probed by the X-ray beam and each element covers $4 \mu \mathrm{m}$ of the sample. Further, prism $\langle 11 \overline{2} 0\rangle$, basal $\langle 11 \overline{2} 0\rangle$, and pyramidal $\langle 11 \overline{2} 3\rangle$ slip systems were assumed to be active.

In order to examine the possibility of determining active slip systems through linking CPFE to 3DXRD, for the second model a "redundant" slip system was intentionally added to the CPFE model. This model will be referred as S4Pa for which all of the model set-up was identical to that of S4, except for active slip systems and their CRSS values; that is, it was assumed that prism $\langle 11 \overline{2} 0\rangle$, basal $\langle 11 \overline{2} 0\rangle$, and pyramidal $\langle 11 \overline{2} 3\rangle$, and pyramidal $\langle 11 \overline{2} 0\rangle$ slip systems were active with the CRSS values of 95,127 , 287 , and $95 \mathrm{MPa}$, respectively. CRSS values were extracted such that the macroscopic stress-strain curve can be replicated while the ratio between the two main active slip systems, i.e. basal and prism, stays the same as the one used for the Model S4. A comparison between the macroscopic stress -strain curves calculated by each model is shown in Fig. 1c. Further, the CPFE calculated relative activities for each slip system at the onset of plasticity and applied strain of $1.2 \%$ for the Models S4 and S4Pa are shown in Fig. 2. Relative activities are calculated based on the shear strain that each slip system accommodates (see Eq. 11). As expected, most of the plastic deformation for the model S4 is accommodate by prism (1-3) and basal (3-6) systems whereas for the model S4Pa, prism (1-3), basal (4-6), and pyramidal $<\mathrm{a}>(7-12)$ are all active. The results presented in this paper for model S4Pa are for the case where the ratio of the CRSS for basal and prism slip systems is the same as that of model S4, however, we have tested other ratios, yet our conclusions did not change.

Measured orientations at preload were assigned to each grain, i.e. all of the IPs of each grain had the same orientation initially, but as a result of grain-grain interaction, each IP will develop a unique orientation as well as elastic strain when the model is loaded. These orientations and strains for each grain were then used to simulate diffraction peaks. To elucidate, the calibrated global parameters of the 3D-XRD experiment was used to substitute the parameters of Eq. 1 to Eq. 8, i.e., sample to detector distance of $374.5 \mathrm{~mm}$, pixel size of $50 \mu \mathrm{m}$, and the beam energy of $78.39 \mathrm{keV}$ were used. It is worth mentioning that the pixel size is required to convert the position of a peak (Eq. 8) to a pixel number on the $2048 \times 2048$ area detector. The centre of the detector was also moved to the position determined during calibration of the experimental set-up. The 3D-XRD global parameters were calibrated using a standard Ceria sample during the experiment. The second step in the modelling of the peaks was to import the CPFE calculated elastic strains and updated Euler angles into the code used for updating the reciprocal lattice vectors using Eq. 2 (details of calculating lattice strains are given in Abdolvand et al [41]). Once $\vec{r}_{h k l}^{s}$ for a given ( $h k l$ ) plane was determined, it was possible to solve Eq. 6 and determine the position of the peak on the detector. 


\section{Fig. 2 Here}

\section{Results and discussion}

Since the macroscopic applied strain was quite small, grains with large plastic deformation in the CPFE simulation were selected to increase the likelihood that strong peak broadening could be observed. The criteria for selecting a grain was:

(a) The selected grain should not have any element at the surface of the model so that the effects of the boundary conditions can be reduced.

(b) The selected grain should have maximum slip activity per IP, i.e., plastic deformation should not just localize at the grain boundaries, instead, it should be observed across entire grain. Also, without condition (b), big grains will automatically be selected as more IPs generally means more plasticity. Maximum plasticity per IP is the implemented criteria here.

(c) The selected grain should have at least 950 IPs assigned to it. This will ensure that small grains are not selected so that the effects of deformation heterogeneity can be studied.

(d) It should be possible to track the selected grain from preload to unload in the experiment so that the evolution of the grain orientation and diffraction peaks measured experimentally can be possible for the entire experiment.

On average, 100 diffraction peaks were assigned to each grain; it is neither possible nor informative to attempt to visualise all 100 diffraction peaks. The following criteria was used to select particular peak of a selected grain [36]:

(A) The selected peak should not overlap with any other peaks in any of the $\theta, \eta$, and $\omega$ domains.

(B) The selected peak must fulfil the diffraction condition for the $\omega$ belonging to the range of [-224 to -115$]$ and [-45 to 45]. With this condition, the peaks close to the edge of $\omega$-domain will be neglected so that the spread of selected peak over this domain can be studied.

(C) Similar to (B), the selected peak must have $\eta$ belonging to the range [8 to172] and [188 to 352] as the peaks outside this range tend to stretch over the $\omega$-domain (see Eq. 7).

(D) Conditions (A) to (C) should be valid across all of the loading steps.

(E) It should be possible to track the selected peak over all of the loading steps.

In what follows, some of the grains and the peaks that fulfil the conditions mentioned above are analysed and discussed. Four different categories are considered: grains with maximum prism, basal, pyramidal $<\mathrm{c}+\mathrm{a}>$, and pyramidal $<\mathrm{a}>$ slip activity. Also, the numerical results provided in this section are all outputs of the Model S4 unless otherwise stated.

\subsection{Grains with maximum prism activity}

As it is shown in Fig. 1d, most of grains have their c-axis along the global-x direction and since the tensile load was applied along the global-z direction, prism is the predominant slip system in most of the grains. The CPFE results for the Model S4 indicates that the grain 5382 was one of the plastically softest grains in the simulated volume. As it is shown in Table 1, the cumulative plastic shear accommodated on all of the prism planes of this grain is more than $1 \%$ at the applied strain of $1.2 \%$; These are the average values calculated for the 3696 IPs assigned to this grain,. The measured Euler angles of this grain at the preload and applied strain of $1.2 \%$ are given in Table 2 . With using these Euler angles, it was revealed that the misorientation between the basal plane normal (BPN) and the loading direction $(\beta)$ was $88.69^{\circ}$ and that of the closest prism plane normal (PPN) was $9.4^{\circ}$ with the other two PPNs at $50.7^{\circ}$ and $69.3^{\circ}$ which reinforces the high level of prism activity. It is shown in Table 
1 that the only active slip system for this grain is prism as the activities of basal and pyramidal $<\mathrm{c}+\mathrm{a}>$ are negligible.

Table 1: Shear accommodated on each slip system of Grain 5382 at $\varepsilon_{\text {applied }}=1.2 \%$

\begin{tabular}{l|c|c|c|c}
\hline & Number of IPs & \multicolumn{3}{|c}{ Shear on slip system $\left(\mathbf{x ~ 1 0} \mathbf{1 0}^{-3}\right)$} \\
\hline & & Prism & Basal & Pyramidal $<\mathbf{c}+\mathbf{a}>$ \\
\hline CPFE Model S4 & 3696 & 10.73 & 0.2 & $8 \mathrm{E}-4$ \\
\hline
\end{tabular}

The calculated and measured stresses for the grain 5382 in the three macroscopic reference axes are shown in Fig 3a. These results are given in the global coordinate were 11, 22, and 33, coincide with the global $\mathrm{x}, \mathrm{y}$, and $\mathrm{z}$, respectively. The CPFE simulations show that this grain yields early and has $\sigma_{33}$ well below the macroscopic average as it is soft. This is further observed in the distribution of the stress across the grain; in all of the three main directions, the range of the IP values is very much expanded from onset of plasticity to the applied strain of $1.2 \%$. Further, the plastic deformation induced a vivid stress heterogeneity within the grain at the unload comparing to the preload; $300 \mathrm{MPa}$ variation of $\sigma_{33}$ at unload as opposed to $200 \mathrm{MPa}$ at the preload.

Since the effects of the elastic strains and lattice rotations on the shape of the diffraction peaks will be discussed, as an example, the evolution of elastic strain within this grain is also illustrated in Fig. $3 \mathrm{~b}$. While non-zero residual elastic strain exists at the preload and stays almost the same at unload, strain heterogeneity increases significantly across all of the steps. This is a result of plastic deformation as well as local grain-grain interaction. For instance, along the y-axis the grain averaged stress $\left(\sigma_{22}\right)$ becomes tensile while the strain $\left(\varepsilon_{22}\right)$ shows a contraction indicating significant constraint of the natural Poisson contraction by the neighbouring grains.

One of the main outputs of the CPFE simulations is the updated crystal orientation and elastic strain for each IP at each loading step. This could be extracted as the UMAT code used was capable of calculating the induced grain rotation resulting from large deformation. In Fig. 3c, the distributions of lattice rotations calculated for IPs within grain 5382 are shown. The misorientation and rotations are calculated with respect to the preload, the step at which all of the IPs of a grain have the same orientation from the initial grain averaged 3D-XRD preload measurement. Also, the propensity on the vertical axis refers to the total number of IPs that have their misorientation within a given range. It is shown that while the total misorientation and the rotation of PPNs vary up to $1.6^{\circ}$, the rotation of BPNs is quite small. This is because slip on prism planes mostly rotates PPNs around the crystal c-axis. This is further confirmed by the 3D-XRD results where average rotations of $0.45^{\circ}$ and $0.85^{\circ}$ were measured for BPNs and PPNs, respectively. Both of these values fall into the range of calculated results from CPFE simulation.

\section{Fig 3 Here}

The evolution of two diffraction peaks are presented in detail. The first diffraction peak is $(\overline{2} 0.0)$ which

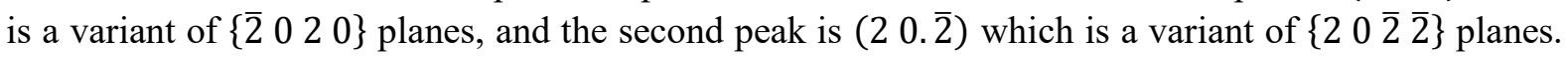
The simulated peak dispersion in terms of $\theta, \omega$, and $\eta$ are shown in Fig. $3 \mathrm{~d}$ and 3e. As described in section 2, the atomic spacing and cryptographic orientation are two of the main parameters that determine the position of a peak on a detector. The former is affected by elastic strain whereas the latter is affected by the elastic lattice rotation. Four cases are studied:

I. Measured orientation at preload was used for simulating diffraction peaks with no lattice strain.

II. Elastic strain calculated at each IP at applied strain of $1.2 \%$ was used for simulating diffraction peaks. This case is called Stretch (S). 
III. Elastic lattice rotation calculated at each IP at the applied strain of $1.2 \%$ was used for simulating diffraction peaks. This case is called Rotation (R).

IV. Elastic lattice rotation and elastic strain calculated at each IP at the applied strain of $1.2 \%$ were used for simulating diffraction peaks. This case is called Stretch and Rotation (RS).

For each case the updated Euler angles and lattice spacings were used to substitute parameters of Eq. 2 from which a new set of $(\theta, \eta, \omega)$ can be calculated for each IP. These results for the peak $(\overline{2} 0.0)$ is shown in Fig. 3d. As expected, since there is no lattice rotation for the case (R), only one $\theta$ is calculated, whereas a very narrow distribution for the both $\mathrm{S}$ and RS cases are observed. In contrast, a much wider distribution is observed for the both $\eta$ and $\omega$ domains. These two domains are mainly affected by the lattice rotations; this is the reason why for the (S) case just a peak with no distribution is observed.

The corresponding measured and simulated $(\overline{2} 0.0)$ peaks are shown in Fig. $4 \mathrm{a}$ to $4 \mathrm{~h}$. The evolution of the $(\overline{2} 0.0)$ peak measured by 3D-XRD experiment is shown in Fig 4a to 4c. A red circle is drawn around this peak to distinguish it from peaks caused by other grains. The effects of plastic deformation of the sample on the smearing of this peak is very clear as it belongs to one of the softest grains in the simulated volume. Further, the rotation calculated for this peak is shown in Fig. 4d, which reveals a large rotation with respect to the preload step. Similar to Fig 3c, for calculating plane rotations, updated Euler angles at the applied strain of $1.2 \%$ were extracted from CPFE simulation and used to calculate the misorientation of the plane normals with that of the preload step. The rotation calculated for $(\overline{2} 0.0)$ plane ranges from 0 to $1.8^{\circ}$ with the average of 0.65 while the measured average rotation was $0.85^{\circ}$ which falls into calculated range.

In Fig. $4 \mathrm{e}$ to $4 \mathrm{~h}$, the simulated ( $\overline{2} 0.0$ ) peaks for the four cases described above are shown. For the first case (I), the orientation of the grain at the preload was used and it was assumed that the lattice is strain free. For such case, diffraction peak will be a single spot, hence, the size of the diffraction spot in Fig. $4 \mathrm{e}$ is exaggerated for clarity. The position of the peak is very well captured in comparison to the measured peak at the preload (Fig 4a). In Fig. 4f, it is shown that the effects of lattice strain on the peak shape and position is negligible. The peak is just slightly spread in the $\theta$ - domain with hardly any influence on the calculated $\eta$ and $\omega$. On the other hand, a large smearing is observed in the $\eta$-domain when the effect of grain rotation is considered; it is shown in Fig. $4 \mathrm{~g}$ that rotation results in smearing of the peak over more than 20 pixels on the detector which is very close to the measured case shown in Fig. 4c. This peak is also broaden in the $\theta$ - domain if the effects of both stretch and rotation are considered (Fig. 4h).

\section{Fig. 4 Here}

As mentioned in section 2, it was assumed that the X-ray beam was absolutely monochromatic. In a real experiment, there will be a small variation in the X-ray beam energy that eventually results in a broader peak. The grain size and shape are two other factors that affect the peak shape. For bigger grains, diffraction from different grain sections would appear around the central diffraction spot, assuming no orientation variation. These two factors were neglected in our simulation of diffraction peaks and are the reason for the differences observed between Fig. 4a and 4e. For instance, the estimated size of the grain 5382 is $79 \mu \mathrm{m}$ that corresponds to 1.6 pixels on the detector. Further, in our simulation, it was assumed that the detector is perfect with no distortion. The effects of detector distortion were taken into account during the analysis of the measured patterns, however, the measured peaks shown in this paper are all raw measured peaks. It is also worth mentioning that the effects of detector tilts were considered in our peak simulations but it revealed to have minor effects for the current test set-up[39].

The evolution of the $(20 . \overline{2})$ is shown in Fig. $4 \mathrm{i}-4 \mathrm{p}$. Similar to the previous case, this peak smears progressively as more strain is applied to the grain, however, less broadening is observed at the applied strain of $1.2 \%$ compared to the $(\overline{2} 0.0)$ peak. Please note that to provide a like-to-like comparison, a box of $100 \times 100$ pixels is used to present all of the simulated and measured peaks in this paper. To 
understand the reason why less broadening is observed for $(20 . \overline{2})$, the rotations of this peak calculated for all of the grain IPs are shown in Fig. 41. In comparison to the $(\overline{2} 0.0)$ peak, a narrower distribution is calculated with a smaller average. This is also captured in the simulated peaks. For instance, it is shown in Fig. 3e that $\eta$ and $\omega$ for $(20 . \overline{2})$ peak varies over the range of 1.2 and 1.8 degrees, respectively, whereas those of $(\overline{2} 0.0)$ peak varies over the range of 2 and 2.1 degrees. Further, we showed earlier that the c-axis of the grain 5382 is oriented toward the global-x axis (almost perpendicular to the loading direction). Slip on the prism plane rotate the crystal around the c-axis, hence, the most affected crystallographic planes, would be those without any c- component, eg. ( $\overline{2} 0.0)$.

\section{Fig. 5 Here}

The simulated positions of $(20 . \overline{2})$ planes using different assumptions are shown in Fig. $4 \mathrm{~m}-4 \mathrm{p}$. In comparison to $(\overline{2} 0.0)$ peak, the effect of elastic strain on the peak broadening in the $\theta$ - domain is slightly greater (Fig. 4n). This is also shown in Fig. 3e where a wider variation is calculated for $\theta$. Further, a narrower peak distribution is captured for the $\eta$-domain (see Fig. $4 p$ ) while in $\omega$ the smearing is more evident (fig 3e).

In Figs. $4 \mathrm{e}$ and $4 \mathrm{~m}$, the measured orientation at the preload were used for simulating diffraction pattern assuming that crystal lattice is strain free. Heat treatment of CPZr can induce orientation and strain variation, even at the preload. To investigate the effects of thermal residual stresses, the CPFE calculated strains and orientations were used for simulating diffraction peaks. The results for $(\overline{2} 00)$ and $\left(\begin{array}{lll}2 & 0 & \overline{2}\end{array}\right)$ peaks are shown in Fig. $5 \mathrm{a}$ and $5 \mathrm{~b}$ where a minor smearing is observed. This smearing is negligible comparing to those measured or calculated at the unload step. In Fig. $5 \mathrm{c}$ and $5 \mathrm{~d}$, the calculated strain and orientation variation at the unload are used for simulating the diffraction peaks. It is shown that even $1.2 \%$ of straining can induce large peak smearing. This is further confirmed by the peaks measured in the 3D-XRD experiment (Fig. 5e and 5f).

In Fig. $3 \mathrm{~d}$ and $3 \mathrm{e}$, it was shown that strain and orientation variations within a grain result in peak smearing mainly in the $\eta$ and $\omega$ domains. In Figs. 4 and 5, the $\omega=40.5^{\circ}$ and $\omega=-3^{\circ}$ were used for extracting the experimental and measured $(\overline{2} 0.0)$ and $\left(\begin{array}{ll}2 & 0 . \overline{2}\end{array}\right)$ diffraction peaks, respectively, although these peaks appear in other $\omega$ steps, as well. These are the $\omega$-steps that the selected peaks had highest visibility, or intensity, at the preload and just for the sake of consistency, we used the same $\omega$-steps across all of the loading steps. As an example, the spread of these two peaks in the $\eta$ and $\omega$ domains combined are shown in Fig. 5g and 5h. The CPFE calculated strain and orientation at $1.2 \%$ strain were used for simulating smearing in $\eta$ and $\omega$ domains. It is clear that the selected $\omega$ s have the highest contribution from diffracting IPs. 
Table 2: Comparison between results of CPFE and 3DXRD for Grain 5382

\begin{tabular}{|c|c|c|c|c|c|c|c|c|c|c|c|c|c|}
\hline & & \multicolumn{3}{|c|}{ Euler Angles } & \multicolumn{3}{|c|}{ State of stress (MPa) } & \multicolumn{3}{|c|}{ Reflection $(20 . \overline{2})$} & \multicolumn{3}{|c|}{ Reflection $(\overline{2} 0.0)$} \\
\hline \multirow{3}{*}{ Preload } & & $\varphi_{1}$ & $\varphi$ & $\varphi_{2}$ & $\sigma_{33}$ & $\sigma_{22}$ & $\sigma_{11}$ & $\boldsymbol{\theta}$ & $\eta$ & $\omega$ & $\boldsymbol{\theta}$ & $\eta$ & $\omega$ \\
\hline & 3D-XRD & 278.82 & 271.31 & 69.31 & $-19 \pm 4$ & $26 \pm 4$ & $45 \pm 8$ & 7.36 & 146.58 & 40.36 & 6.47 & -20.49 & -3.17 \\
\hline & CPFE & & & & -54 & 8.8 & 67 & 7.36 & 146.55 & 40.43 & 6.47 & -20.49 & -3.12 \\
\hline \multirow{2}{*}{$\varepsilon=1.2 \%$} & 3D-XRD & 279.25 & 271.18 & 70.13 & $209 \pm 13$ & $48 \pm 16$ & $-55 \pm 23$ & 7.35 & 146.86 & 41.09 & 6.45 & -19.65 & -3.05 \\
\hline & CPFE & & & & 202 & 32 & -18 & \multicolumn{3}{|c|}{ See Fig. 3d } & \multicolumn{3}{|c|}{ See Fig. 3e } \\
\hline
\end{tabular}

Table 3: Comparison between results of CPFE and 3DXRD for Grain 7241

\begin{tabular}{|c|c|c|c|c|c|c|c|c|c|c|c|c|c|}
\hline \\
\hline & & \multicolumn{3}{|c|}{ Euler Angles } & \multicolumn{3}{|c|}{ State of stress (MPa) } & \multicolumn{3}{|c|}{ Reflection (1 $\overline{1.4})$} & \multicolumn{3}{|c|}{ Reflection $(0 \overline{1.3})$} \\
\hline & & $\varphi_{1}$ & $\varphi$ & $\varphi_{2}$ & $\sigma_{33}$ & $\sigma_{22}$ & $\sigma_{11}$ & $\boldsymbol{\theta}$ & $\boldsymbol{\eta}$ & $\boldsymbol{\omega}$ & $\boldsymbol{\theta}$ & $\eta$ & $\boldsymbol{\omega}$ \\
\hline \multirow{2}{*}{ Preload } & 3D-XRD & 231.22 & 329.94 & 169.67 & $83 \pm 13$ & $30 \pm 14$ & $21 \pm 20$ & 7.73 & 189.68 & 21.67 & 6.18 & 159.8 & 43.4 \\
\hline & CPFE & & & & 62 & -18 & -53 & 7.74 & 189.71 & 21.48 & 6.18 & 159.85 & 43.4 \\
\hline \multirow{2}{*}{$\varepsilon=1.2 \%$} & 3D-XRD & 231.64 & 329.69 & 169.65 & $340 \pm 20$ & $16 \pm 23$ & $41 \pm 31$ & 7.71 & 190 & 19.04 & 6.17 & 159.63 & 44.15 \\
\hline & CPFE & & & & 318 & -14 & -1 & \multicolumn{3}{|c|}{ See Fig. 5c } & & & \\
\hline
\end{tabular}

Table 4: Comparison between results of CPFE and 3DXRD for Grain 3432

\begin{tabular}{|c|c|c|c|c|c|c|c|c|c|c|c|c|c|}
\hline & & \multicolumn{3}{|c|}{ Euler Angles } & \multicolumn{3}{|c|}{ State of stress (MPa) } & \multicolumn{3}{|c|}{ Reflection (00.2) } & \multicolumn{3}{|c|}{ Reflection $(\overline{\mathbf{1}} 1 . \overline{3})$} \\
\hline & & $\varphi_{1}$ & $\varphi$ & $\varphi_{2}$ & $\sigma_{33}$ & $\sigma_{22}$ & $\sigma_{11}$ & $\theta$ & $\eta$ & $\omega$ & $\boldsymbol{\theta}$ & $\eta$ & $\omega$ \\
\hline \multirow{2}{*}{ Preload } & 3D-XRD & 48.81 & 349.58 & 311.34 & $-23 \pm 14$ & $2 \pm 18$ & $-57 \pm 25$ & 3.51 & 349.6 & -40.63 & 6.18 & 201.69 & -13.78 \\
\hline & CPFE & & & & 107 & -27 & -50 & 3.51 & 349.73 & -39.05 & 6.18 & 201.67 & -13.80 \\
\hline \multirow{2}{*}{$\varepsilon=1.2 \%$} & 3D-XRD & 50.41 & 350.0 & 310.21 & $305 \pm 4$ & $-24 \pm 5$ & $-51 \pm 7$ & 3.50 & 350.05 & -40.48 & 6.17 & 202.17 & -14.36 \\
\hline & CPFE & & & & 388 & -47 & -72 & \multicolumn{3}{|c|}{ See Fig. 7c } & & & \\
\hline
\end{tabular}




\subsection{Grains with maximum basal activity}

The second predominant slip system of CPZr is $<\mathrm{a}>$ basal (see Fig. 2). Since it is easier to activate prism slip, finding a grain that deforms solely by basal slip and fulfils the conditions given in section 4 was challenging. The grain 7241 was selected to study as the ratio of the basal to prism activities is 3.6 which is one of the highest between all of the grains that were simulated. In CPFE simulations, 1000 IPs were assigned to this grain where the contribution from basal slip was $0.553 \%$ (see Table 5). This is the average shear for all of the IPs assigned to this grain. The orientation of this grain in the polycrystal is rather different (see Table 3); the misorientation of the c-axis of the grain with the global $x, y$, and $z$ directions are 67, 72, and 30 degrees, respectively, where the global z-axis coincides with the loading direction. Further, the misorientation of the PPN with the three global directions are respectively 24, 23 , and 60 degrees. With these orientations, it is expected that the grain 7241 show a mixture of both soft and hard behaviour.

Table 5: Shear accommodated on each slip system of Grain 7241 at $\varepsilon_{\text {applied }}=1.2 \%$

\begin{tabular}{c|c|ccc}
\hline & Number of IPs & \multicolumn{3}{c}{ Shear on slip system $\left(\mathbf{x ~ 1 0} \mathbf{1 0}^{-3}\right)$} \\
\hline & & Prism & Basal & Pyramidal $<\mathbf{c}+\mathbf{a}>$ \\
\hline CPFE Model S4 & 1000 & 1.54 & 5.53 & $1.5 \mathrm{E}-2$ \\
\hline
\end{tabular}

The evolution of stress for grain 7241 is shown in Fig. 6a. A very good agreement between CPFE and 3D-XRD results achieved, and the three measured normal stresses fall within the variation zones generated by the IPs assigned to the grain. Further, while the thermal residual $\sigma_{33}$ at the preload vary over a range of $250 \mathrm{MPa}$, at the applied strain of $1.2 \%$, a wider variation zone of $400 \mathrm{MPa}$ was calculated for this grain. The measured and calculated average stress for this grain are also given in Table 3 . Both CPFE and 3D-XRD reveal a positive residual stress in the loading direction at the preload which increases to $340 \mathrm{MPa}$ at the applied strain of $1.2 \%$.

In Fig 6b, the distribution of misorientation of each IP with respect to their original orientation at the preload as well as the rotation of BPNs and PPNs are shown. Interestingly, all of the IPs of this grain experienced at least $0.2^{\circ}$ misorientation which is also seen in the rotation of BPNs. In contrast to the grain 5382 for which a bigger rotation of PPNs were calculated, for the basal slip grain 7241 BPNs rotate more than PPNs. Moreover, our CPFE simulation indicates that PPNs rotate $0.38^{\circ}$, on average, which is close to $0.36^{\circ}$ measured during the experiment. This rotation is a result of slip on both basal and prism systems.

Two of the diffraction peaks that fulfil conditions (A) to (E) are $(1 \overline{1} . \overline{4})$ and $(0 \overline{1} . \overline{3})$. The measured and calculated $\theta, \eta$, and $\omega$ at which these two peaks fulfil diffraction condition are shown in Table 3 . For the preload step, the measured orientation with no lattice stretch was used in the simulation. However, for the applied strain of $1.2 \%$, different cases were examined results of which for the peak $(1 \overline{1} . \overline{4})$ are shown in Fig. 6c. In agreement with the discussion given in section 5.1, the cases (R) and (RS) have the highest influence on the distribution of $\eta$, and $\omega$ whereas lattice stretch (S) affects $\theta$. It is shown in Fig $6 \mathrm{c}$ that $(1 \overline{1} . \overline{4})$ peak must appear over a wider range of $\omega$. The RS case indicates that $\omega$ ranges from $14^{\circ}$ to $20^{\circ}$, given $0.25^{\circ}$ of $\Delta \omega$, the simulation indicate that this peak should appear in about 24 diffraction images. Further investigation of experimental results showed that $(1 \overline{1} . \overline{4})$ peak appeared in $18 \omega$ steps which is close to the simulation. The reason for the small discrepancy between CPFE and experiment is that about 40 IPs diffract for the $\omega$ less than $15^{\circ}$ or higher than $19.5^{\circ}$ which means that the diffraction spot on the detector should be with minimum visibility or even lower than the sensitivity of the detector. 


\section{Fig. 6 Here}

In Fig. 7 the measured and simulated $(1 \overline{1} . \overline{4})$ peaks are illustrated. Experimental results shown in Fig $7 \mathrm{a}-7 \mathrm{c}$ indicate that plastic deformation resulted in a clear peak broadening which progressively smears with more deformation applied. In Fig. 7e, the simulated position of the diffraction peak on the detector for the strain free lattice with the orientation that was measured at the preload is illustrated. It can be seen that a good estimation of the peak position in the horizontal (y) axis is achieved, however, the position of the peak in the vertical direction (z) is off. The reason for such deviation is that detector distortion increases with moving toward the edge of the detector, i.e., good agreement for peak position is achieved in the y direction because the diffraction peak is closer to the middle of the detector; on the other hand, in the $\mathrm{z}$ direction, this peak appears very close to the edge of the detector. This peak is the 15 th peak in the patterns and it was particularly chosen to show the reason why only the first 12 diffraction rings were used for analysing 3D-XRD data. For the first 12 rings, the effects of such distortions are much smaller.

In Fig. 7f, it is shown that the lattice strain resulted in peak broadening in the z-direction; this expected as z-direction for this peak, nearly coincides with radial direction. The effects of rotation on the peak broadening is shown in Fig $7 \mathrm{~g}$. CPFE simulation revealed that $(1 \overline{1} . \overline{4})$ peak rotates about $0.84^{\circ}$ degrees with respect to the preload with $0.4^{\circ}$ of variation about the average. Such variation resulted in broadening over 15 pixels. The addition of lattice stretch to the previous case has just broadened the peak in the vertical direction (Fig. 7h). Interestingly, the direction of peak broadening from CPFE simulation is very much in agreement with the one measured experimentally (Fig. 7c).

\section{Fig 7 Here}

\subsection{Grain with maximum pyramidal $\langle c+a>$ activity}

In Fig. 1d, it is shown that a few number of crystals have their c-axis oriented toward the loading direction. To accommodate deformation along the crystal c-axis it is necessary to activate one of the pyramidal slip systems or tensile twinning. Post-test EBSD map of the sample indicated that twining was not active, particularly since the applied strain is quite small (see supplementary file). The grain 3432 was selected for analysis as it had one of the highest pyramidal $<\mathrm{c}+\mathrm{a}>$ activity in the simulation. In Table 6, it is shown that 1360 IPs were assigned to this grain were the total shear accommodated on the pyramidal $<\mathrm{c}+\mathrm{a}>$ slip systems were $0.27 \mathrm{E}-3$ at the applied strain of $1.2 \%$. More information regarding this grain is given in Table 4. Using the measured orientation, it was revealed that the misorientation between the BPN and the loading direction is $10.4^{\circ}$ which is expected to be low; also, the misorientation between the PPN and the loading direction for the three PPNs are $79.8^{\circ}, 83.13^{\circ}$, and $86.68^{\circ}$.

Table 6: Shear accommodated on each slip system of Grain 3432 at $\varepsilon_{\text {applied }}=1.2 \%$

\begin{tabular}{c|c|ccc}
\hline & Number of IPs & \multicolumn{3}{|c}{ Shear on slip system $\left(\mathbf{x ~ 1 0} \mathbf{1 0}^{-3}\right)$} \\
\hline & & Prism & Basal & Pyramidal $<\mathbf{c}+\mathbf{a}>$ \\
\hline CPFE Model S4 & 1360 & 0.252 & 0.595 & 0.27 \\
\hline
\end{tabular}

The evolution of the calculated and measured stress for grain 3432 is shown in Fig. 8a. Generally all of the measured stresses fall into the variation zone calculated at IPs of the model. However, the average measured stress in the loading direction $\left(\sigma_{33}\right)$ is lower than the calculated one. Since the difference between the measured and calculated values for $\sigma_{33}$ is relatively constant across all of the loading steps, 
it can be speculated that there might have been an offset in the stress measured at the preload; the fact that this grain is mainly deformed elastically reinforces such speculation. One of the factors that affects such an offset is the single crystal parameters that were fitted for all of the grains in the aggregate which may have not worked perfectly for this particular grain. Another source of discrepancy could be in the steps that were used in the modelling. In the first step of the CPFE modelling, the polycrystal were cooled down from $600{ }^{\circ} \mathrm{C}$ to the room temperature to account for the residual stresses that develop during annealing the sample. In our CPFE code, the effect of stress relaxation that may happen at elevated temperature is neglected. Hence, thermal expansions or contraction result solely in the stress build-up that never relaxes with another source. Such assumption can also contribute to this difference.

\section{Fig. 8 Here}

The total misorientation and rotation of BPNs and PPNs with respect to the preload are shown in Fig. $8 \mathrm{~b}$. As expected, the lattice rotations are small comparing to the other two cases studied in the previous sections, although there are some IPs that experience more than $1^{\circ}$ rotation of BPNs; this is solely a result of grain-grain interaction. The effects of such rotations are also shown in the peaks presented in Fig. 9 and Table 4. The $\left(\begin{array}{ll}0 & 0.2\end{array}\right)$ peak is selected for further investigation. This is one of the most affected peaks as slip on pyramidal $<\mathrm{c}+\mathrm{a}>$ results in the tilt of crystal c-axis. The calculated rotation of $\left(\begin{array}{lll}0 & 0.2\end{array}\right)$ peak is shown in Fig. $8 \mathrm{~b}$; for convenience this is also shown in Fig. 9d. On average, $0.28^{\circ}$ rotation with respect to the preload is calculated for this peak which varies up to $1.4^{\circ}$ for a small number of IPs. The effects of such rotation is clearly seen in the $\omega$-domain which varies between $\sim-43^{\circ}$ to $\sim-37^{\circ}$. This means that the $\left(\begin{array}{ll}0 & 0.2\end{array}\right)$ peaks should appear in more than 24 diffraction images which is close to the 20 diffraction images that was experimentally observed. On the other hand, $\theta$ and $\eta$ do not show much variations. This is also observed in the peak shapes that experimentally and numerically captured (see Fig. 9a to $9 \mathrm{~h}$ ). Minimum variation in the peak shape is experimentally observed from preload to the applied strain of $1.2 \%$ (Fig. 9a to 9c). The simulated peak position, which is based on the measured orientation at the preload, is very much consistent with the experimentally measured one; it is shown that if the effects of crystal rotation and stretch are considered, small variation in the peak position in the both $\theta$ and $\eta$ domains will be achieved (see Fig. 9e to 9h) which is also consistent with the experimental observation.

Fig. 9 Here

\subsection{Grains with maximum pyramidal $<a>$ activity}

In the previous sections, the effects of lattice rotation and stretch on the positions and shapes of the diffraction peaks were investigated. It was shown that lattice rotations affect $\eta$ and $\omega$ significantly and lattice strains slightly affect $\theta$. We use these two properties to study deformation mechanisms of CPZr at room temperature; more specifically, in this section, it is tried to determine if pyramidal $<\mathrm{a}>\operatorname{slip}$ is active at significant levels. Pyramidal $<\mathrm{a}>$ slip has been proposed as a slip mode in $\mathrm{Zr}$ by various authors [eg 48, Liu et al, Mat Sci Eng A. 719, (2018), 147-154, Lebensohn, Sanchez \& Pochettino, Scripta Metall., 30 (1994), 481-486, Philippe et al, Acta Metall. Mater., 43 (1995), 1619-1635, Tome', Lebensohn \& Kocks, Acta Metall. Mater. 39, (1991) 2667, Xu, Holt \& Daymond, J. Nucl. Mater., 373 (2008), 217-225]. As Xu et al note although simulations including pyramidal $<$ a $>$ slip fit to macroscopic stress-strain, and even lattice strain data reasonably well, there is a lack of direct evidence for its occurrence at room temperature. 
On the modelling side, all of the results provided so far were extracted from the model S4 for which prism, basal, and pyramidal $<\mathrm{c}+\mathrm{a}\rangle$ were the active slip systems. In this section, these results are compared to those from the model $\mathrm{S} 4 \mathrm{~Pa}$, for which pyramidal $<\mathrm{a}>$ is also active. The calculated lattice strain and rotation from each model were used to simulate the peak positions of all of the peaks that could potentially be detected in the experiment while fall into the first 12 diffraction rings, i.e., peaks close to the edge of the detector are ignored. Subroutines were then developed to cross correlate the simulated peaks with the experimentally captured ones. At this point, two methods were used to examine the possible activity of pyramidal $<\mathrm{a}>$ slip system; in the first method (M1), since each peak appears in several diffraction images, the centre of mass of each peak from the experiment and the models were determined and compared. To quantify the difference between the simulated and measured values, a parameter is introduced which uses Eq. 16 to determine $\overline{E r}$ :

$$
\overline{E r}=\sum_{i=1}^{n o . P e a k s}\left[\left(\bar{\theta}_{i}^{s}-\bar{\theta}_{i}^{e}\right)^{2}+\left(\bar{\eta}_{i}^{s}-\bar{\eta}_{i}^{e}\right)^{2}+\left(\bar{\omega}_{i}^{s}-\bar{\omega}_{i}^{e}\right)^{2}\right]
$$

where $i$ represent the $i^{\text {th }}$ diffraction peak that was possible to find in both simulation and 3D-XRD experiment, $\bar{\theta}_{i}^{s}, \bar{\eta}_{i}^{S}$, and $\bar{\omega}_{i}^{S}$ represent the simulated mean position for the $\mathrm{i}^{\text {th }}$ peak in the $\theta, \eta$, and $\omega$ domains, respectively, and $\bar{\theta}_{i}^{e}, \bar{\eta}_{i}^{e}$, and $\bar{\omega}_{i}^{e}$ represent the corresponding measured mean position in the $3 \mathrm{D}-\mathrm{XRD}$ experiment- both sets are given in degrees. $\overline{E r}$ represents the cumulative error of determining diffraction peak positions for all of the cross-correlated peaks. It is worth mentioning that the centre of the mass of diffraction peaks for the experiment is one of the outputs of the ImageD11 software.

The second method (M2) that was used for investigating the activity of the pyramidal $<a>$ slip system was to determine the most affected peaks by such slip activity. For this purpose, for each grain that was investigated, the measured orientation from the preload step was used to determine the normal to the planes of the peaks that were collected in the experiment. The calculated Euler angles extracted from the models S4 and S4Pa after 1.2\% straining were used to determine the normal to the planes of the same peaks. The misorientation between the plane normals at the applied strain of $1.2 \%$ and preload were subsequently calculated for both S4 and S4Pa models using calculated orientation at each IP. The average misorientation for each plane from all of the IPs was then calculated. At this stage, the plane normal of each peak has two misorientations associated with it, one from S4 model and one from S4Pa model. The absolute difference between these two misorientation for each peak was then calculated. From the final step, it was possible to determine which peaks were affected the most from the activity of pyramidal $<\mathrm{a}>$ slip system. Once the most affected peaks for a grain were determined, it was possible to compare the calculated rotation from CPFE model and those measured experimentally and hence comment on the activity of the pyramidal $<\mathrm{a}>$ slip system. The mathematical formulation for this method is given in Eq. 17.

$$
\begin{aligned}
& \vec{r}_{h k l}^{1}=R_{G}^{\text {Preload }} \vec{r}_{h k l}^{c} \\
& \vec{r}_{h k l}^{2, I P}=R_{G}^{S 4, I P} \vec{r}_{h k l}^{c} \\
& \vec{r}_{h k l}^{3, I P}=R_{G}^{S 4 P, I P} \vec{r}_{h k l}^{c} \\
& P=\max \left\{\left|\overline{m i s o r\left(\vec{r}_{h k l}^{1}, \vec{r}_{h k l}^{2, I P}\right)}-\overline{m i s o r}\left(\vec{r}_{h k l}^{1}, \vec{r}_{h k l}^{3, I P}\right)\right|\right\}
\end{aligned}
$$

where $R_{G}^{\text {Preload }}$ represent the rotation matrix of the grain $\mathrm{G}$ at the preload step, $R_{G}^{S 4, I P}$ and $R_{G}^{S 4 P, I P}$ are the calculated rotation matrixes at each IPs of the same grain using the results of the models S4 and S4Pa after $1.2 \%$ straining, respectively, $\vec{r}_{h k l}^{c}$ represent the lattice vector of the $(h k l)$ plane in the crystal coordinate, and $P$ represent the peaks that were significantly affected by the activity of pyramidal $<\mathrm{a}>$ slip system. In principle, in the method M1, the deviation of simulated patterns from the measured ones 
is investigated whereas in the method $\mathrm{M} 2$, the misorientation of each plane normal with respect to its initial configuration is investigated.

Two grains with the highest $<\mathrm{a}>$ pyramidal slip activity indicated by model S4Pa were firstly selected. These grains also fulfilled the conditions provided in (a)-(d). The first of these was grain 7414 for which the CPFE results of models S4 and S4Pa are shown in Table 7. It is shown that 960 IPs were assigned to this grain where in the model $\mathrm{S} 4$, prism and basal were the two dominant deformation mechanisms; however, in model S4Pa, basal and pyramidal $<\mathrm{a}>$ were the two dominant mechanisms. The activity of pyramidal $<\mathrm{c}+\mathrm{a}>$ for both models is negligible. The second selected grain for further analysis is the grain 3467 which has 1008 IPs. Higher $<a>$ Pyramidal activity is calculated for this grain in model S4Pa which prism and basal both being active as well. The measured and calculated average stress for both grains are provided in Table 8. Results of preload for both models are identical as thermal residual strains are almost entirely elastic. At the applied strain of $1.2 \%$, not much difference is observed in the results of the CPFE models in comparison to the 3D-XRD measured values. This indicates that elastic strain is not as sensitive as lattice rotation in the accurate determination of the simulated peak positions.

Table 7: Shear accommodated on each slip system of Grain 7414 and 3467 at $\varepsilon_{\text {applied }}=1.2 \%$

\begin{tabular}{|c|c|c|c|c|c|c|c|c|}
\hline \multirow[b]{2}{*}{ Grain } & \multirow[b]{2}{*}{ CPFE Model } & \multirow[b]{2}{*}{$\begin{array}{c}\text { Number } \\
\text { of IPs }\end{array}$} & \multicolumn{4}{|c|}{ Shear on slip system $\left(\times \mathbf{1 0}^{-3}\right)$} & \multirow[b]{2}{*}{$\begin{array}{c}\text { Number of } \\
\text { simulated peaks }\end{array}$} & \multirow[b]{2}{*}{$\begin{array}{l}\text { Cumulative error in } \\
\text { peak positions }(\overline{E r})\end{array}$} \\
\hline & & & Prism & Basal & $\begin{array}{c}\text { Pyramidal } \\
<\mathbf{a}>\end{array}$ & $\begin{array}{c}\text { Pyramidal } \\
<\mathbf{c}+\mathbf{a}>\end{array}$ & & \\
\hline 7414 & S4 & 960 & 4.35 & 8.9 & 0 & 0.21 & 81 & 60 \\
\hline 7414 & S4Pa & 960 & 0.13 & 5.5 & 3.5 & 0.05 & 81 & 77 \\
\hline 3467 & S4 & 1008 & 9.67 & 5.26 & 0 & 0 & 53 & 74 \\
\hline 3467 & S4Pa & 1008 & 1.58 & 1.4 & 5.46 & 0 & 53 & 145 \\
\hline
\end{tabular}

In Table 7, it is shown that for grain 7414, it was possible to match 81 peaks from experiment to the simulated ones where the cumulative error $\overline{E r}$ calculated for all of the peaks is bigger for the model S4Pa. For grain 3467, it was possible to match 53 peaks, slightly less than grain 7414 , yet much higher error $\overline{E r}$ was calculated for model S4Pa. This means that the simulated peak positions for the Model S4 are closer to the 3D-XRD measured ones and error $\overline{E r}$ calculated is proportional to the activity of $<\mathrm{a}>$ pyramidal slip system. To shed more light on to this, three of the most affected peaks are selected for further analysis, using M2 method. In Fig. 10a, the calculated rotations of the planes ( $\overline{2} 2.0),(\overline{2} 2 . \overline{1})$, $(\overline{2} 2 . \overline{2})$ for grain 7414 are shown. IP values are used for the model results and rotations are given with respect to the preload step. The average measured orientation are also used to calculate the rotation of each peak in the experiment. These results for each peak is given by a single black bar. It is shown that the rotation histograms of the model S4 peak at the values that are much closer to the experimentally measured ones. This trend is valid for all of the analysed peaks. The analysis of the three most affected peaks of grain 3467, peaks (0 2.0), (0 2.1), and ( $\overline{2} 2.1)$ are shown in Fig 10b. In all of these cases, the calculated rotations using the results of the model S4 peak at the values that are much closer to the experimentally measured ones. Hence, both M1 and M2 methods point to the fact that pyramidal $<\mathrm{a}>$ cannot be active at the room temperature.

Table 8: Comparison between results of CPFE and 3DXRD for Grain 7414 and 3467

\begin{tabular}{|c|c|c|c|c|c|c|c|c|}
\hline \multirow[t]{2}{*}{ Grain } & \multirow{2}{*}{\multicolumn{2}{|c|}{ (2) }} & \multicolumn{3}{|c|}{ Euler Angles } & \multicolumn{3}{|c|}{ State of stress (MPa) } \\
\hline & & & $\varphi_{1}$ & $\varphi$ & $\varphi_{2}$ & $\sigma_{33}$ & $\sigma_{22}$ & $\sigma_{11}$ \\
\hline \multirow{3}{*}{7414} & \multirow{3}{*}{ Preload } & 3D-XRD & 138.62 & 325.44 & 238.32 & $30 \pm 21$ & $2 \pm 26$ & $-39 \pm 33$ \\
\hline & & Model S4 & & & & 72 & 25 & -12 \\
\hline & & Model S4Pa & & & & 72 & 25 & -12 \\
\hline \multirow{3}{*}{7414} & \multirow{3}{*}{$\varepsilon=1.2 \%$} & 3D-XRD & 139.29 & 325.31 & 237.82 & $241 \pm 20$ & $-37 \pm 23$ & $1 \pm 31$ \\
\hline & & Model S4 & & & & 295 & -65 & 28 \\
\hline & & Model S4Pa & & & & 280 & -72 & -6 \\
\hline 3467 & Preload & 3D-XRD & 261.14 & 291.93 & 77.87 & -28 & 2.6 & -33 \\
\hline
\end{tabular}




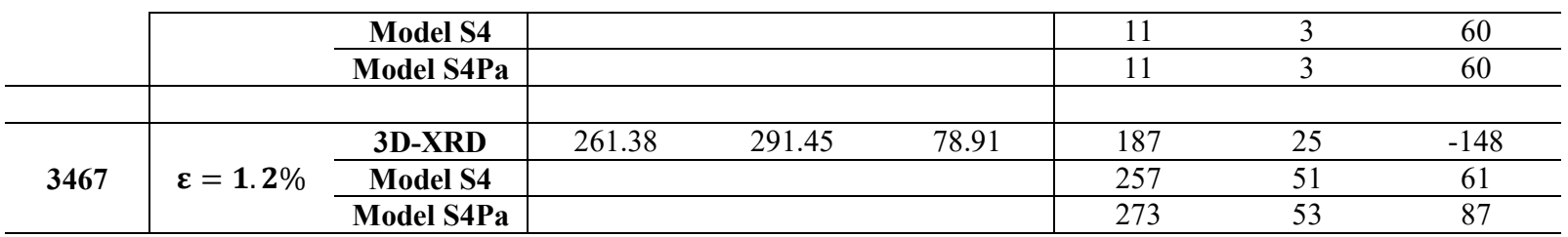

Fig. 10 Here

\section{Summary and Conclusions}

A polycrystalline commercially pure zirconium sample was deformed in uniaxial tension during an insitu 3D-XRD experiment in which a large volume of the sample was probed by the X-ray beam where more than 11000 grains were mapped. A CPFE model was constructed using the grain centroids in a weighted Voronoi tessellation and used to model the initial thermal residual stresses and subsequent deformation to $1.2 \%$ strain and then unload. In this work the focus was on the detailed behaviour of a number of grains selected on the basis of their orientation and expected dominant slip system. Comparisons were made between measured grain averaged stress, or elastic strains and distributions calculated for the equivalent grain in the CPFE simulations. Further, non-uniform lattice rotations were used to simulate expected diffraction peak shapes for comparison to those measured. A model was developed to compare simulated diffraction spots to those measured. The results and analysis presented here have some implications for work in this area and we make the following main points in conclusion:

(i) It was shown that lattice rotations affect peak position and peak smearing more significantly than lattice stretch, so that the peaks smear out around the Debye-Scherrer rings while there is much less broadening across the rings. Such peak smearing was captured in both experiment and simulation.

(ii) Our model for predicting diffraction peak position and shape follows others [49] in treating the IPs as independent diffracting entities with a simple summation of the intensities. This assumption neglects the effects of coherent diffraction from short length-scale strain variations such as those near dislocations which are known to become dominant at large strains. The reasonable quantitative agreement between simulations and experiments shown here suggest that our simplifying assumption holds reasonably well at low strain (and hence low dislocation density).

(iii) For the same microstructure and boundary conditions simulations were undertaken with and without the possibility of $<a>$ pyramidal slip in addition to the $<a>$ prism, $<a>$ basal and $<\mathrm{c}+\mathrm{a}>$ pyramidal systems. The bulk stress-strain response could be well reproduced in either case, and even grain resolved stresses did not provide a clear differentiation between the two models. Lattice rotations, however, did provide clear difference between the two cases with the model omitting $<\mathrm{a}>$ pyramidal slip generating a better match to experiment for both diffraction peak shifts and smearing (extent and direction). It is concluded that $<\mathrm{a}>$ pyramidal slip does not contribute significantly to room temperature deformation in $\mathrm{Zr}$ for the investigated applied strain.

(iv) It was shown that even $1.2 \%$ straining can lead to a significant peak broadening in grains of CPZr polycrystals. Diffraction peaks at preload were compared to those at unload and it was shown that peaks tend to smear in both $\omega$ and $\eta$ domains at unload. This was observed in both simulated and measured peaks.

\section{Acknowledgement}


The authors acknowledge the ESRF for allocating beamtime for experiment MA-2492. Authors are grateful to the Engineering and Physical Sciences Research Council (EP/K034332/1) for financial support through the HexMat program. HA acknowledges the financial support of his Discovery Grant from the Canadian Natural Sciences and Engineering Research Council. He also wishes to thank Oxford Micromechanics group for their contribution during his experiment at the ESRF.

\section{References}

[1] Liang ZY, Huang MX. J Mech Phys Solids 2015;85:128.

[2] Armstrong DEJ, Hardie CD, Gibson JSKL, Bushby AJ, Edmondson PD, Roberts SG. J Nucl Mater 2015;462:374.

[3] Bohnert C, Schmitt NJ, Weygand SM, Kraft O, Schwaiger R. Int J Plast 2016;81:1.

[4] Yang DY, Lim TW, Son Y, Barlat F, Yoon JW. Int J Plast 2011;27:1527.

[5] Guo Y, Schwiedrzik J, Michler J, Maeder X. Acta Mater 2016;120:292.

[6] Zhang J, Kishida K, Inui H. Int J Plast 2017;92:45.

[7] Gong J, Benjamin Britton T, Cuddihy MA, Dunne FPE, Wilkinson AJ. Acta Mater 2015;96:249.

[8] Zhang Z, Jun TS, Britton TB, Dunne FPE. Acta Mater 2016;118:317.

[9] Zhang Z, Jun TS, Britton TB, Dunne FPE. J Mech Phys Solids 2016;95:393.

[10] Jun T-S, Zhang Z, Sernicola G, Dunne FPE, Britton TB. Acta Mater 2016;107:298.

[11] Jun TS, Sernicola G, Dunne FPE, Britton TB. Mater Sci Eng A 2016;649:39.

[12] Maaß R, Van Petegem S, Borca CN, Van Swygenhoven H. Mater Sci Eng A 2009;524:40.

[13] Hofmann F, Keegan S, Korsunsky AM. Mater Lett 2012;89:66.

[14] Davydok A, Jaya BN, Robach O, Ulrich O, Micha JS, Kirchlechner C. Mater Des 2016;108:68.

[15] Zhang C, Li H, Eisenlohr P, Liu W, Boehlert CJ, Crimp MA, Bieler TR. Int J Plast 2015;69:21.

[16] Kirchlechner C, Keckes J, Motz C, Grosinger W, Kapp MW, Micha JS, Ulrich O, Dehm G. Acta Mater 2011;59:5618.

[17] Kirchlechner C, Imrich PJ, LiegI W, Pörnbacher J, Micha JS, Ulrich O, Motz C. Acta Mater 2015;94:69.

[18] Maaß R, Uchic MD. Acta Mater 2012;60:1027.

[19] Hofmann F, Tarleton E, Harder RJ, Phillips NW, Ma P, Clark JN, Robinson IK, Abbey B, Liu W, Beck CE. Nat Publ Gr 2017:1.

[20] Woo W, Balogh L, Ungár T, Choo H, Feng Z. Mater Sci Eng A 2008;498:308.

[21] Weisser MA, Van Petegem S, Cervellino A, Van Swygenhoven H. Int J Plast 2015;66:138.

[22] Benafan O, Noebe RD, Padula SA, Brown DW, Vogel S, Vaidyanathan R. Int J Plast 2014;56:99.

[23] Kanjarla a. K, Lebensohn R a., Balogh L, Tomé CN. Acta Mater 2012;60:3094.

[24] Kim E, Woo W, Heo Y, Seong B, Choi J, Choi S. Int J Plast 2016;79:48. 
[25] Mathis K, Csiszar G, Capek J, Clausen B, Lukas P, Vinogradov A, Agnew SR. Int J Plast 2015;72:127.

[26] Guiglionda G, Borbély A, Driver JH. Acta Mater 2004;52:3413.

[27] Wang L, Li M, Almer J. Acta Mater 2013;62:239.

[28] Zhang X, Li M, Park J-S, Kenesei P, Almer J, Xu C, Stubbins JF. Acta Mater 2017;126:67.

[29] Seymour T, Frankel P, Balogh L, Ungár T, Thompson SP, Jädernäs D, Romero J, Hallstadius L, Daymond MR, Ribárik G, Preuss M. Acta Mater 2017;126:102.

[30] Wong SL, Park JS, Miller MP, Dawson PR. Comput Mater Sci 2013;77:456.

[31] McNelis KP, Dawson PR, Miller MP. J Mech Phys Solids 2012;61:428.

[32] Pagan DC, Miller MP. J Appl Crystallogr 2014;47:887.

[33] Winther G, Wright JP, Schmidt S, Oddershede J. Int J Plast 2017;88:108.

[34] Jakobsen B, Poulsen HF, Lienert U, Pantleon W. Acta Mater 2007;55:3421.

[35] Obstalecki M, Wong SL, Dawson PR, Miller MP. Acta Mater 2014;75:259.

[36] Oddershede J, Wright JP, Beaudoin A, Winther G. Acta Mater 2015;85:301.

[37] Abdolvand H, Wright J, Wilkinson AJ. Nat Commun 2018;9:171.

[38] Abdolvand H, Majkut M, Oddershede J, Wright JP, Daymond MR. Acta Mater 2015;93:246.

[39] Poulsen HF. J Appl Crystallogr 2012;45:1084.

[40] Randle V., Engler O. Introduction to Texture Analysis: Macrotexture, Microtexture, and Orientation Mapping. OPA; 2000.

[41] Abdolvand H, Daymond MR, Mareau C. Int J Plast 2011;27:1721.

[42] Abdolvand H, Daymond MR. J Mech Phys Solids 2013;61:803.

[43] Asaro RJ, Needleman a. Acta Metall 1984;33:923.

[44] Fisher ES, Renken CJ. Phys Rev 1964;135.

[45] Lyckegaard A, Lauridsen EM, Ludwig W, Fonda RW, Poulsen HF. Adv Eng Mater 2011;13:165.

[46] Xu F, Holt R a., Daymond MR. Acta Mater 2008;56:3672.

[47] Xu F, Holt R a., Daymond MR, Rogge RB, Oliver EC. Mater Sci Eng A 2008;488:172.

[48] Brenner R, Béchade JL, Castelnau O, Bacroix B. J Nucl Mater 2002;305:175.

[49] Wong SL, Park JS, Miller MP, Dawson PR. Comput Mater Sci 2013;77:456. 
a.

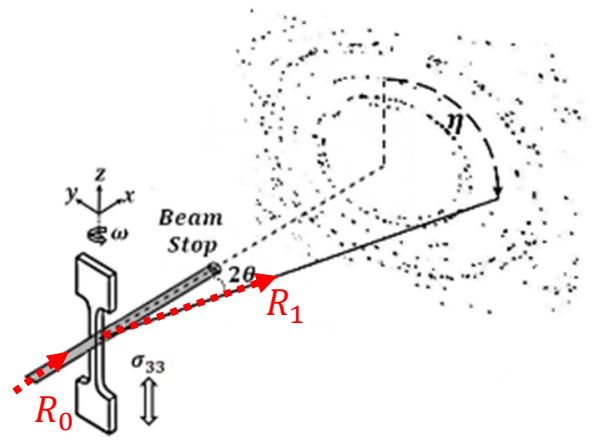

c.

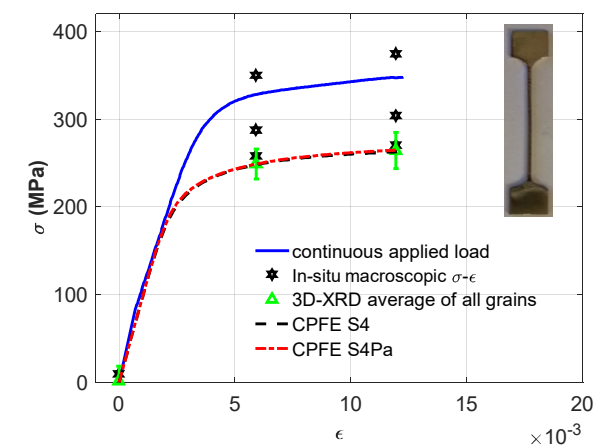

b.

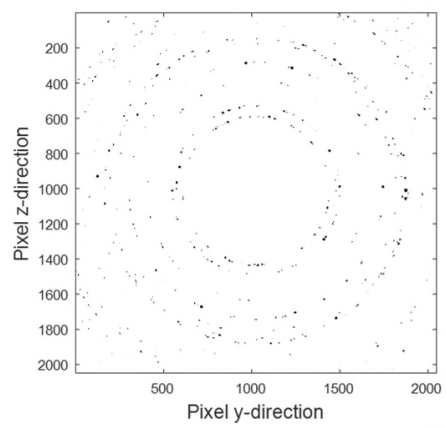

d.

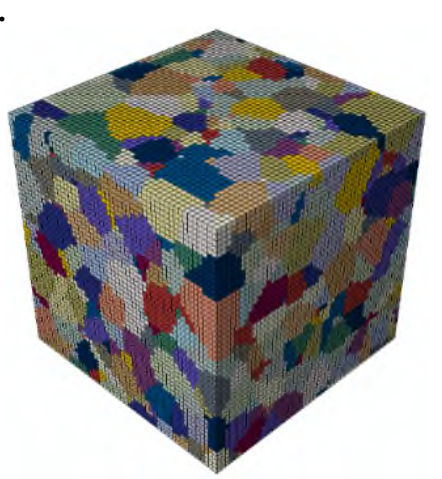

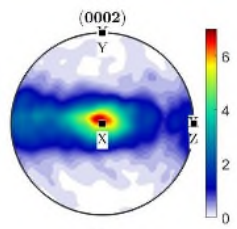

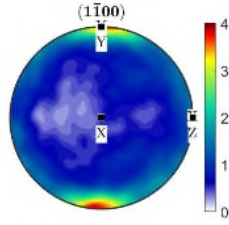

Fig. 1. (a) The schematic of the 3D-XRD experimental set-up. (b) An example of measured diffraction peaks. (c) Comparison between simulated and measured stress-strain curves. (d) The initial measured pole figure of the sample and the corresponding simulated microstructure for finite element modelling. The sample used in the 3DXRD experiment is shown in the right hand side of (c). 
a.

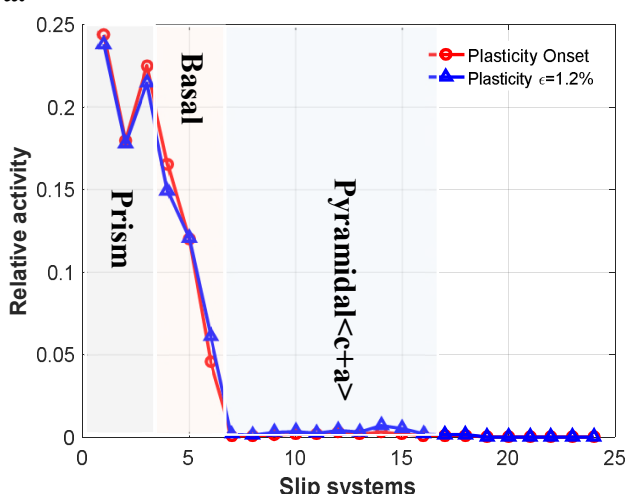

b.

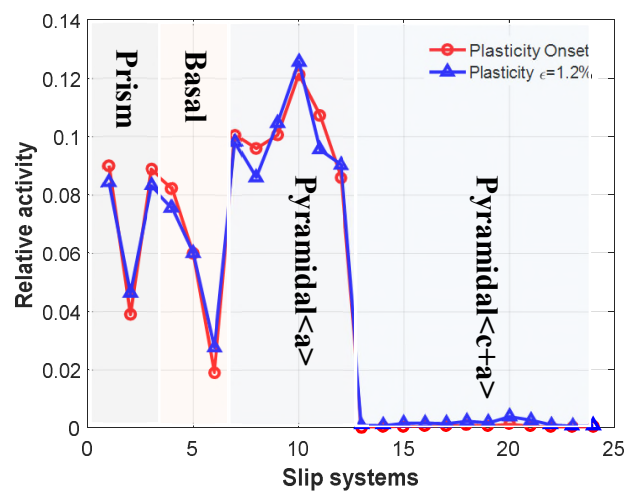

Fig. 2. Relative activities calculated by CPFE modelling at the onset of plasticity and applied strain of $1.2 \%$ for: (a) Model S4 and (b) Model S4Pa. 
a.

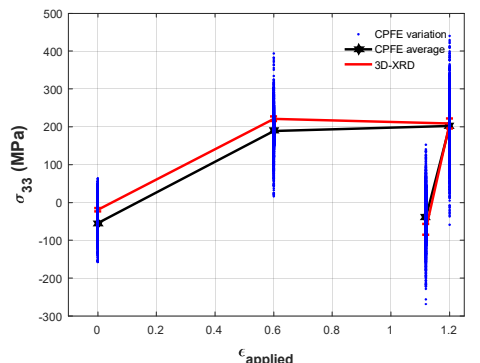

b.

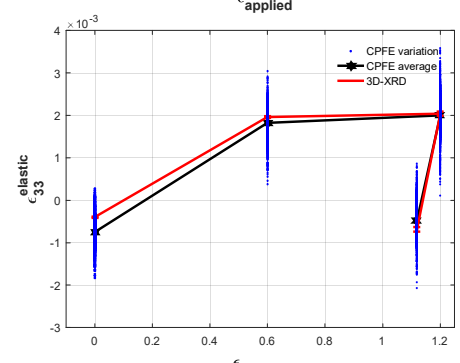

c.

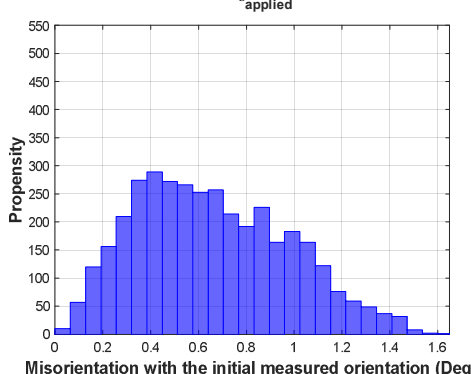

d.

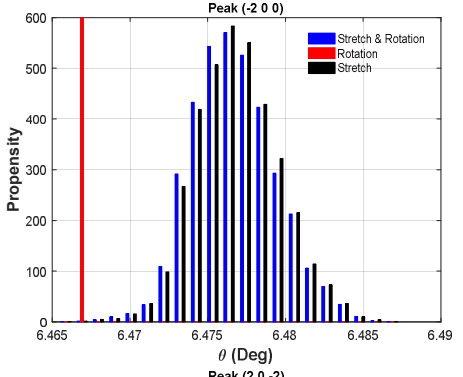

e.

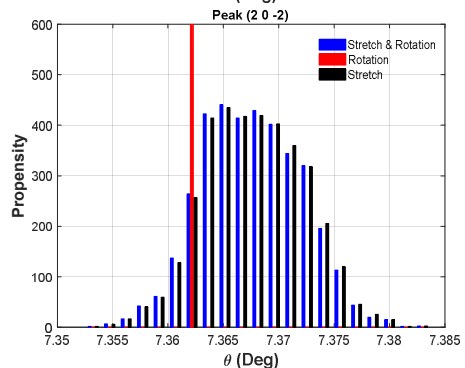

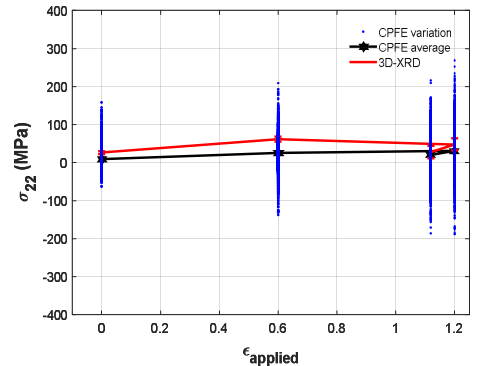
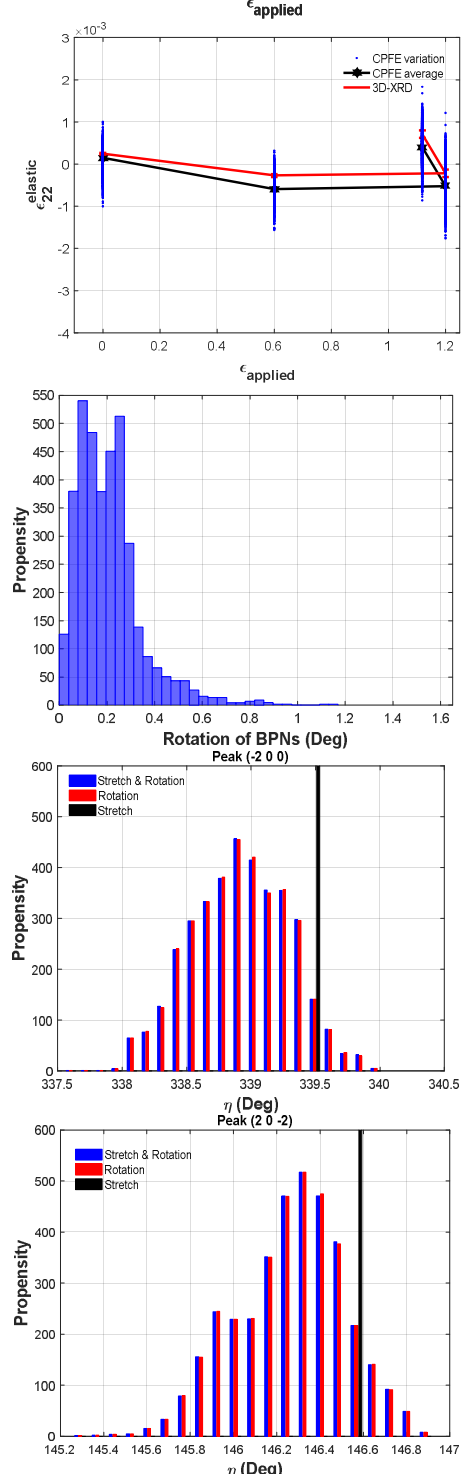
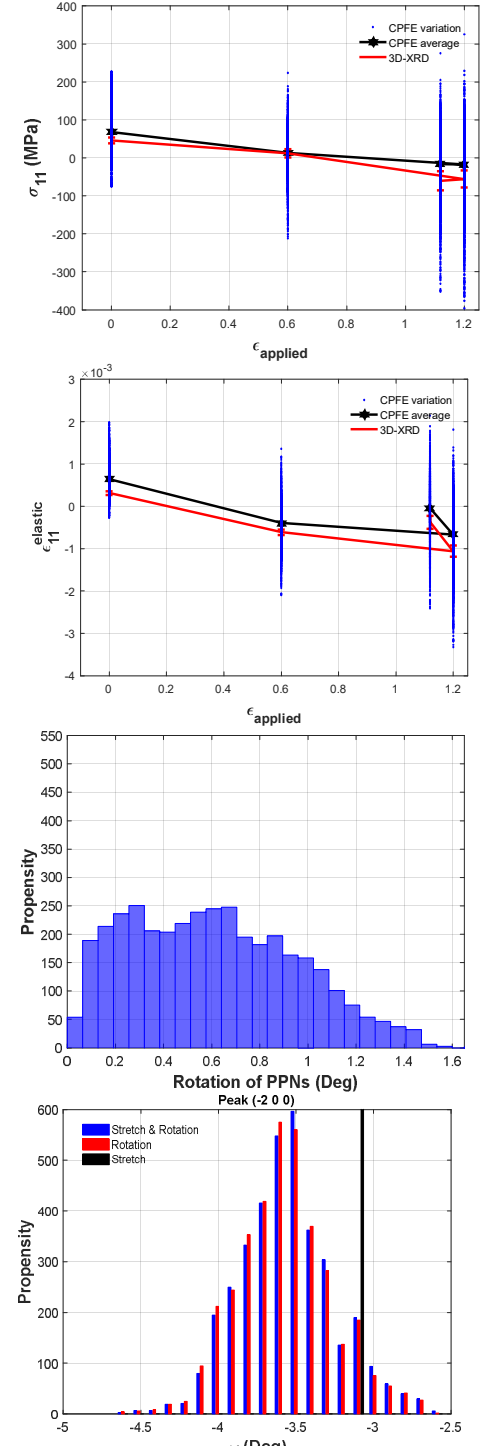
$\underset{\substack{\omega(\mathrm{Deg}) \\ \text { Peak }(20-2)}}{\stackrel{2}{20})}$

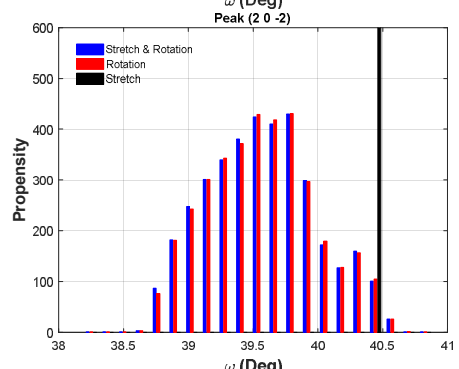

Fig. 3. Results for grain 5382. Comparison between measured and calculated (a) stress and (b) elastic strain in the three main directions. Blue dots are the integration point values, black stars are the CPFE averages for each step and the red line represent the 3DXRD result. Applied strain at unload corresponds to stress-free sample. (c) CPFE results for total misorientation, the rotation of basal plane normals and prism plane normals with respect to those at the preload. Effects of grain stretch and rotation on the position of (d) $(\overline{2} 0.0)$ and (e) ( $20 . \overline{2})$ diffraction peaks. The CPFE integration point values are used to produce (c), (d) and (e). 
a.
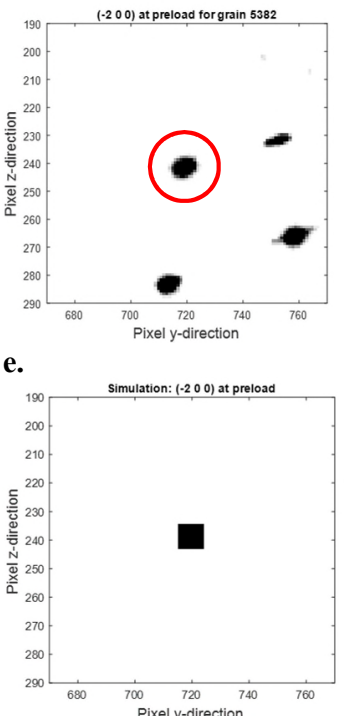

i.

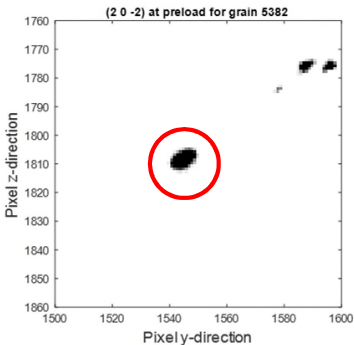

m.

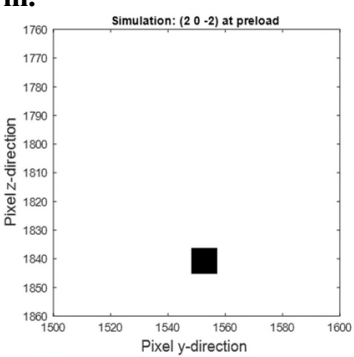

b.
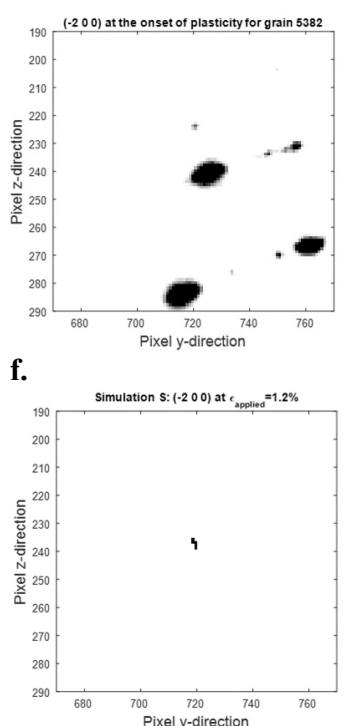

j.

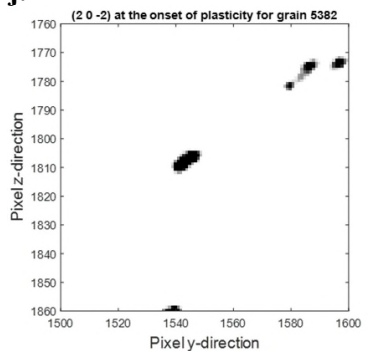

n.

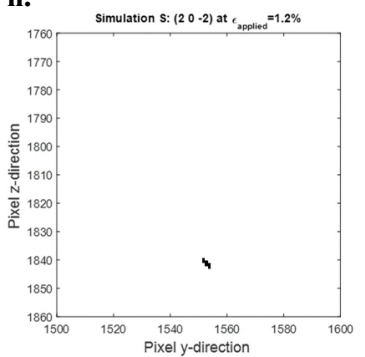

c.

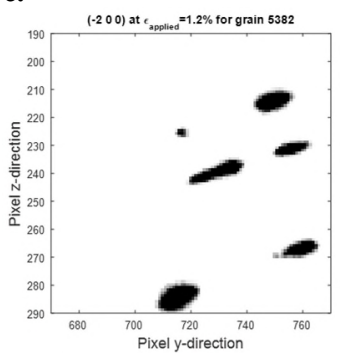

g.

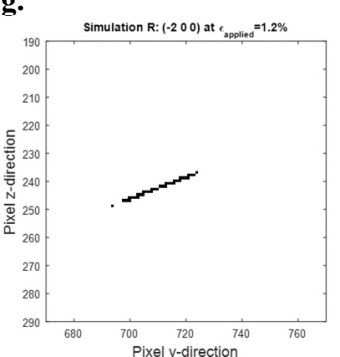

k.

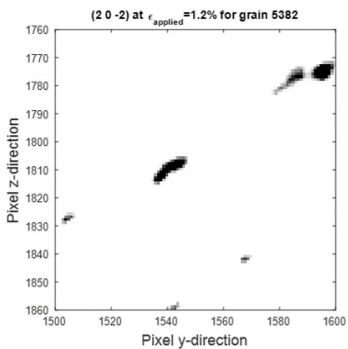

o.

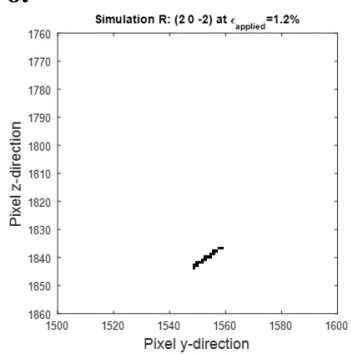

d.

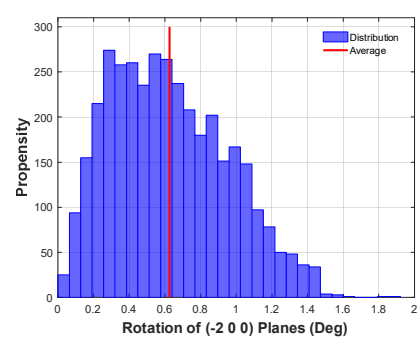

h.

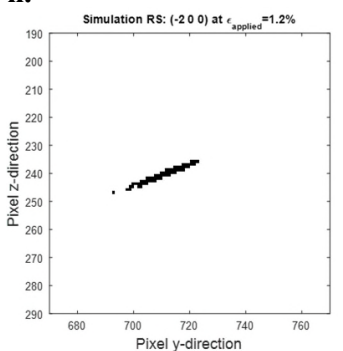

I.

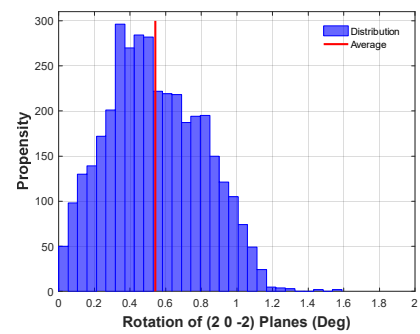

p.

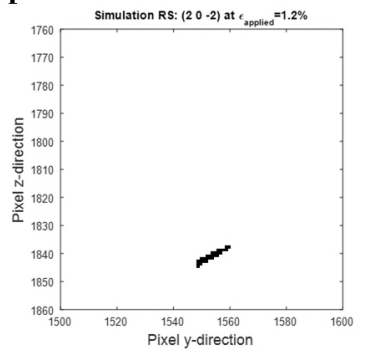

Fig. 4. Comparison between measured and simulated diffraction patterns: measured $(\overline{2} 0.0)$ at (a) preload, (b) onset of plasticity, and (c) $\varepsilon_{\text {appiled }}=1.2 \%$. (d) CPFE calculated rotation of $(\overline{2} 0.0)$ planes at $\varepsilon_{\text {appiled }}=1.2 \%$ with respect to those at preload. (e) Simulated position of $(\overline{2} 0.0)$ peak using the measured Euler angles at preload. Effects of the calculated (f) stretch, (g) rotation, and (h) rotation and stretch on the shape of $(\overline{2} 0.0)$ peak. Measured $\left(\begin{array}{ll}2 & 0 . \overline{2}\end{array}\right)$ at (i) preload, (j) onset of plasticity, and (k) $\varepsilon_{\text {appiled }}=1.2 \%$. (l) CPFE calculated rotation of $(20 . \overline{2})$ planes at $\varepsilon_{\text {appiled }}=1.2 \%$ with respect to those at preload. $(\mathrm{m})$ Simulated position of $(20 . \overline{2})$ peak using the measured Euler angles at the preload. Effects of the calculated (n) stretch, (o) rotation, and (p) rotation and stretch on the shape of $\left(\begin{array}{ll}2 & 0 . \overline{2}\end{array}\right)$ peak. The size of the peaks in (e) and (m) are magnified for clarity. 
a.

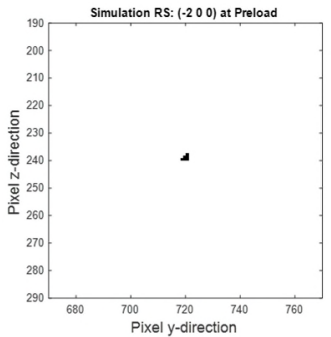

e.

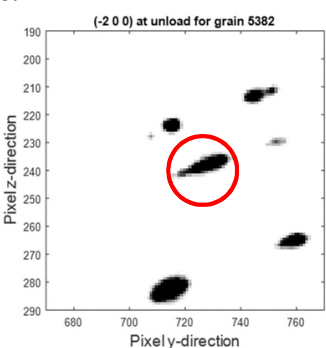

b.
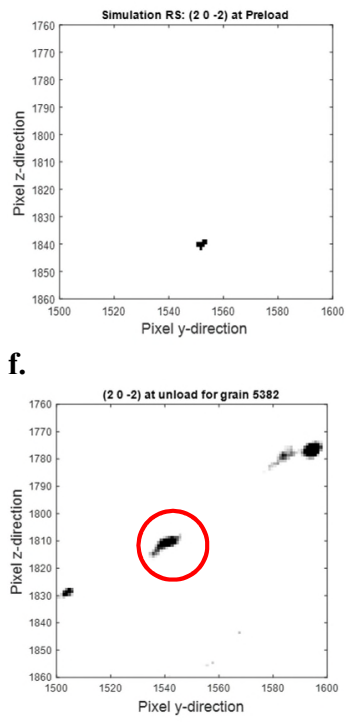

c.

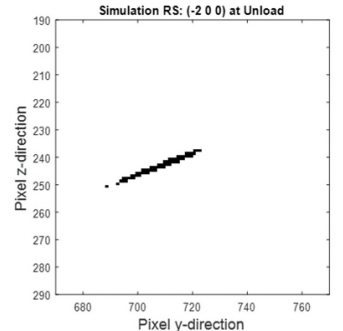

g.

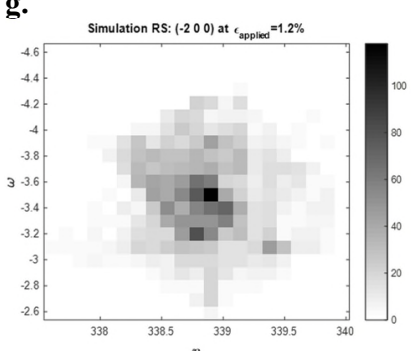

d.
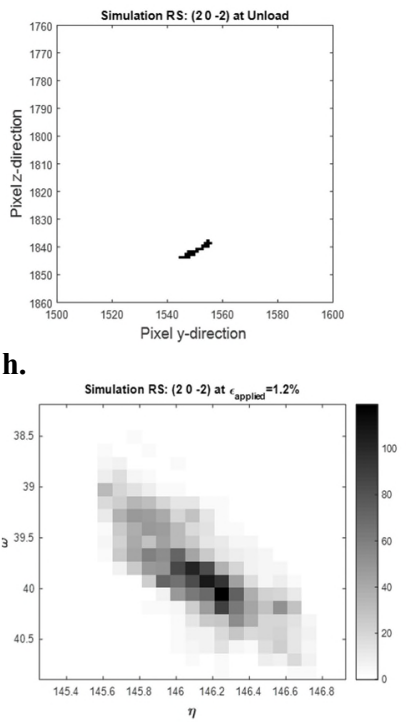

Fig 5. Simulation results at the preload for (a) $(\overline{2} 0.0)$ and (b) $\left(\begin{array}{ll}2 & 0 . \overline{2}\end{array}\right)$ peaks, as well as at unload in (c) and (d), respectively. The measured (e) $(\overline{2} 0.0)$ and (f) $(20 . \overline{2})$ peaks at the unload. The simulation results for smearing of (g) $(\overline{2} 0.0)$ and $(\mathrm{h})(20 . \overline{2})$ peaks at the applied strain of $1.2 \%$. The intensities in (g) and (h) represent the number of integration points that contribute to a diffraction spot. 
a.

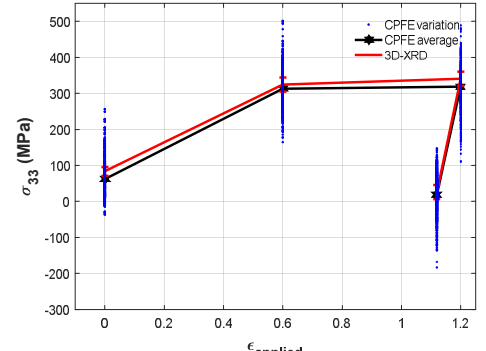

b.
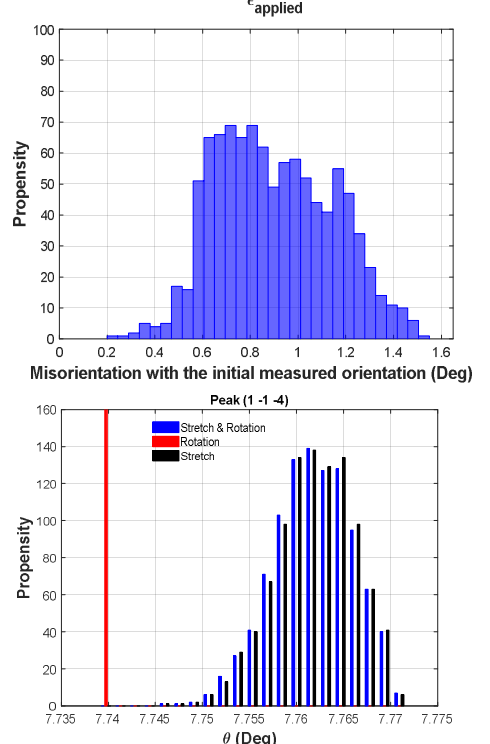
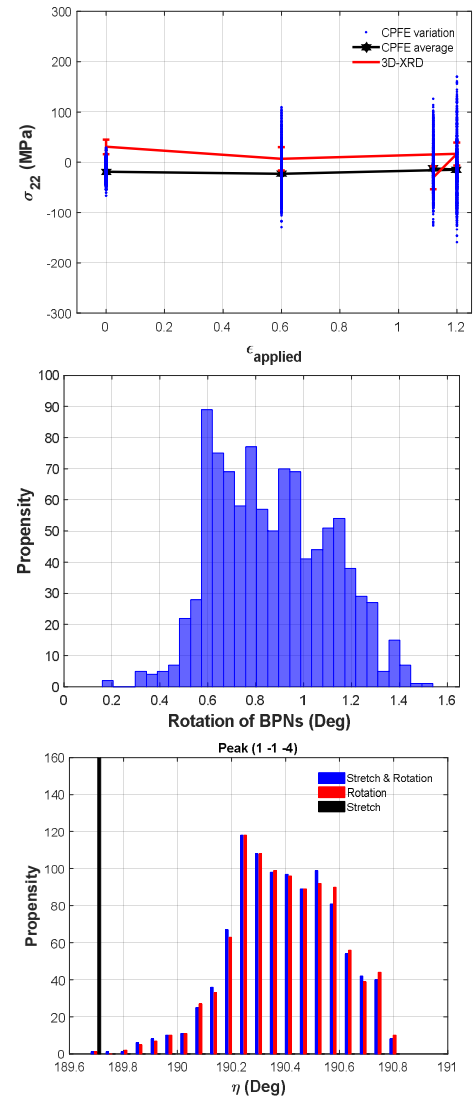
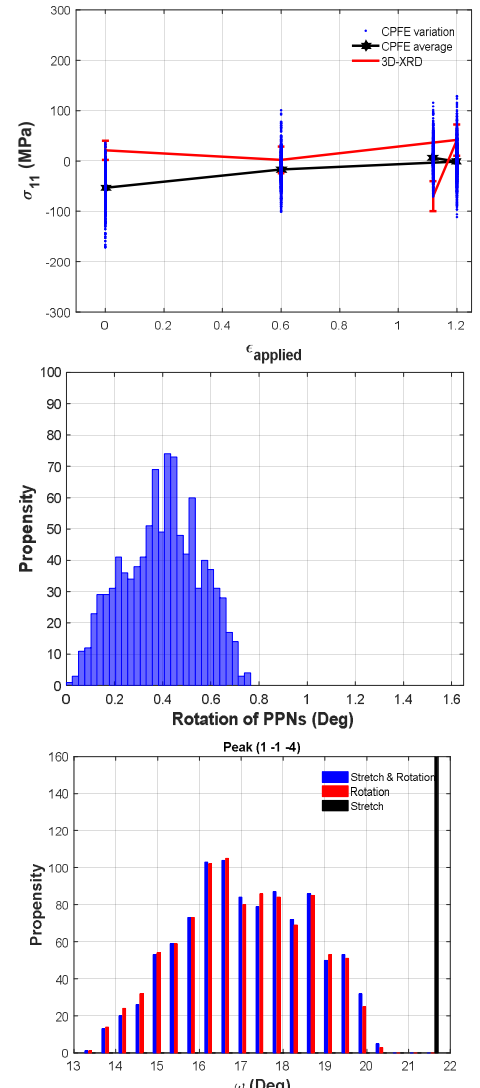

Fig. 6. Results for grain 7241. (a) Comparison between measured and calculated stress in the three main directions. Blue dots are the integration point values, black stars are the CPFE averages for each step and the red line represent the 3DXRD result. Applied strain at unload corresponds to stress-free sample. (b) CPFE results for total misorientation, the rotation of basal plane normals and prism plane normals with respect to those at the preload. (c) Effects of grain stretch and rotation on the position of $(1 \overline{1} . \overline{4})$ peak. The CPFE integration point values are used to produce (b) and (c). 
a.

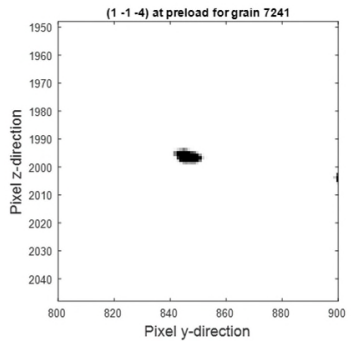

e.

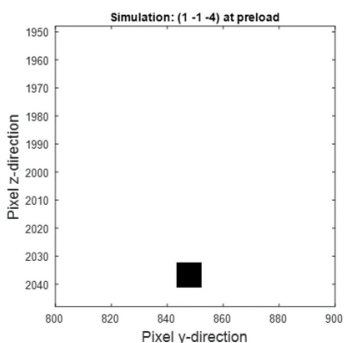

b.

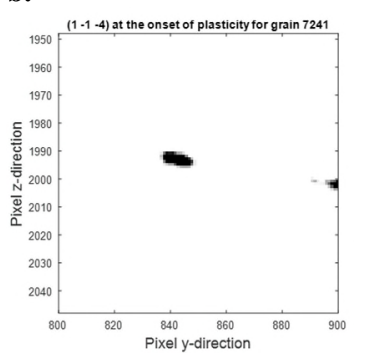

f.

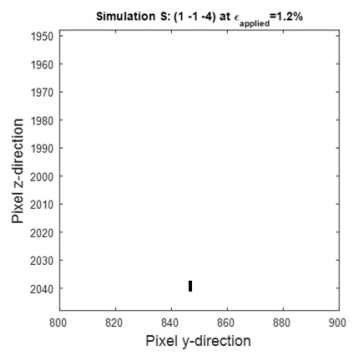

c.

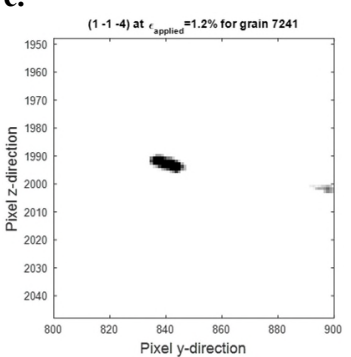

g.

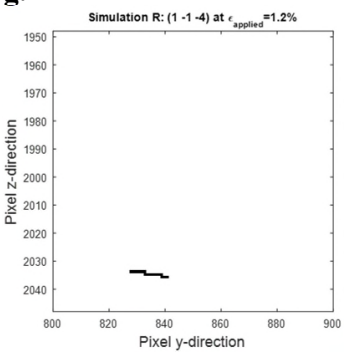

d.

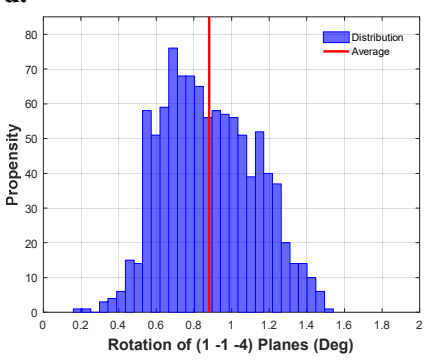

h.

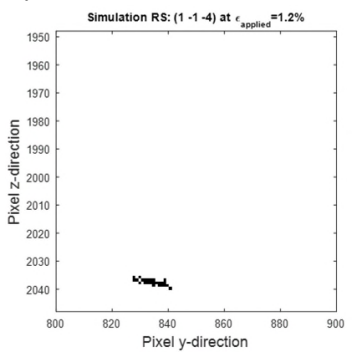

Fig. 7. Comparison between measured and simulated diffraction patterns: measured $(1 \overline{1} . \overline{4})$ at (a) preload, (b) onset of plasticity, and (c) $\varepsilon_{\text {appiled }}=1.2 \%$. (d) CPFE calculated rotation of $(1 \overline{1} . \overline{4})$ planes at $\varepsilon_{\text {appiled }}=1.2 \%$ with respect to those at preload. (e) Simulated position of $\left(\begin{array}{ll}1 & 1 . \\ \hline\end{array}\right)$ peak using the measured Euler angles at preload. Effects of the calculated (f) stretch, $(g)$ rotation, and (h) rotation and stretch on the shape of $(1 \overline{1} . \overline{4})$ peak. The size of the peaks in (e) is magnified for clarity. 
a.

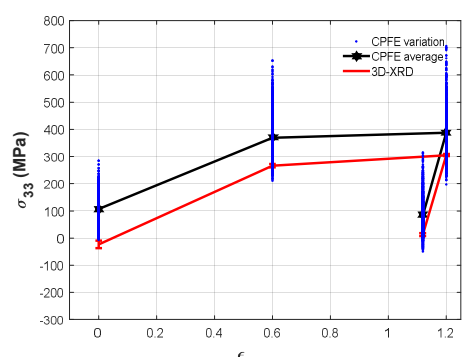

b.

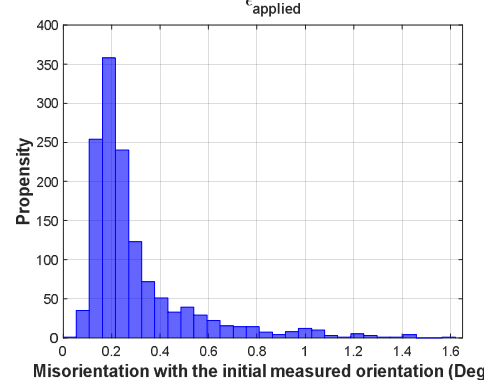

c.

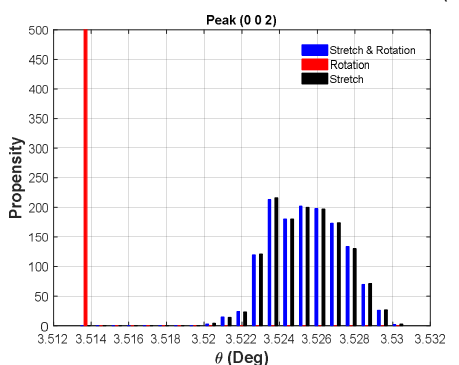

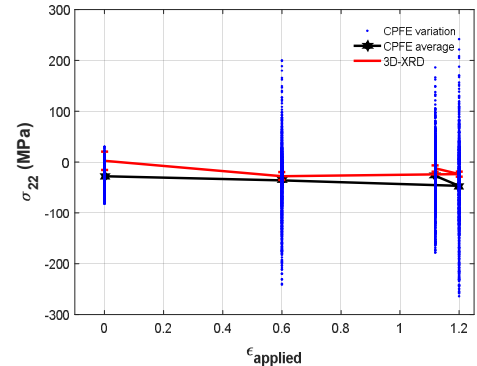
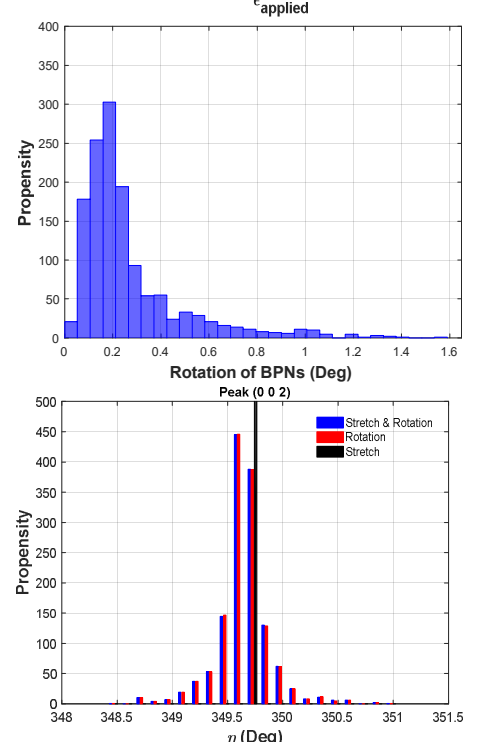
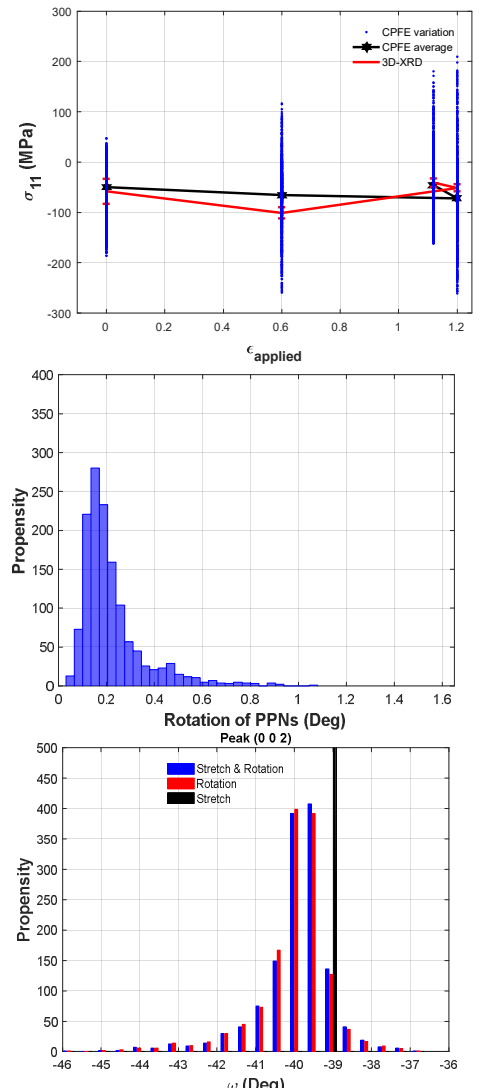

Fig. 8. Results for grain 3432. (a) Comparison between measured and calculated stress in the three main directions. Blue dots are the integration point values, black stars are the CPFE averages for each step and the red line represent the 3DXRD result. Applied strain at unload corresponds to stress-free sample. (b) CPFE results for total misorientation, the rotation of basal plane normals and prism plane normals with respect to those at the preload. (c) Effects of grain stretch and rotation on the position of $\left(\begin{array}{ll}0 & 0.2)\end{array}\right)$ peak. The CPFE integration point values are used to produce (b) and (c). 
a.

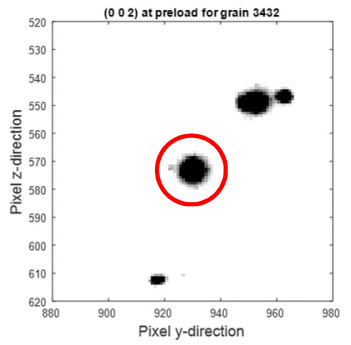

e.

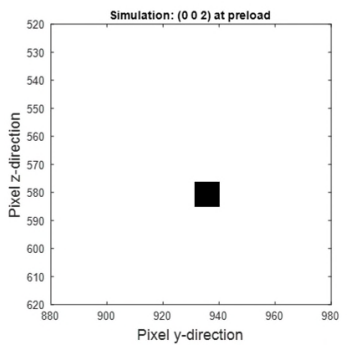

b.

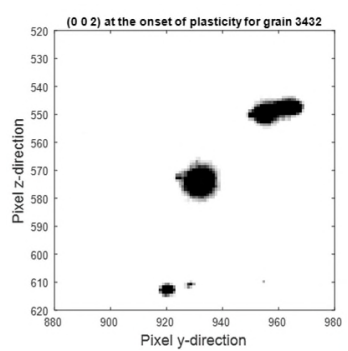

f.

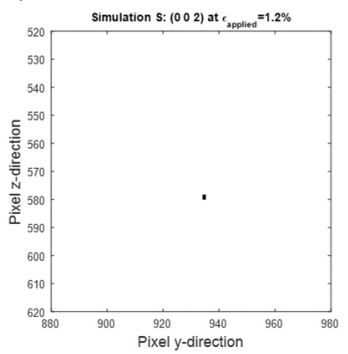

c.

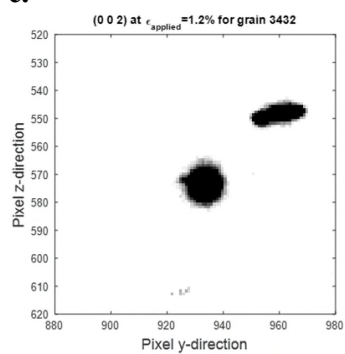

g.

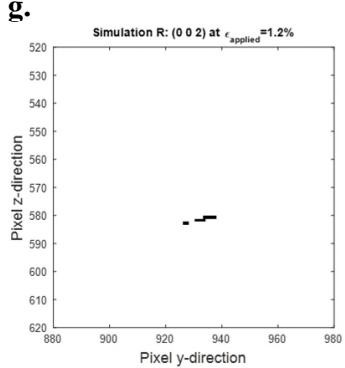

d.

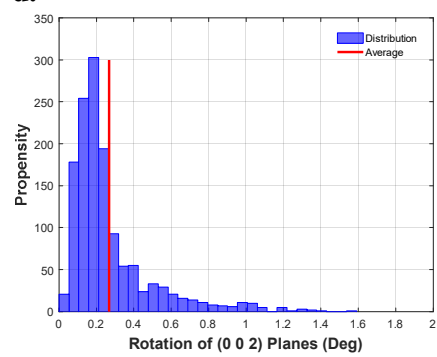

h.

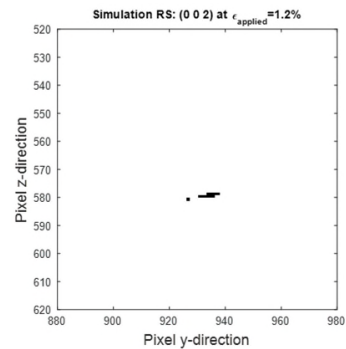

Fig. 9. Comparison between measured and simulated diffraction patterns: measured $\left(\begin{array}{ll}0 & 0.2)\end{array}\right)$ at (a) preload, (b) onset of plasticity, and (c) $\varepsilon_{\text {appiled }}=1.2 \%$. (d) CPFE calculated rotation of $(00.2)$ planes at $\varepsilon_{\text {appiled }}=1.2 \%$ with

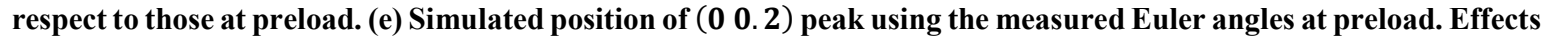

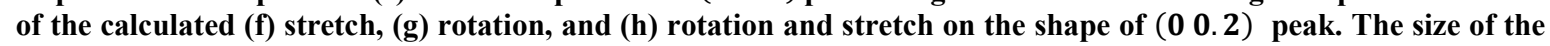
peaks in (e) is magnified for clarity. 
a.

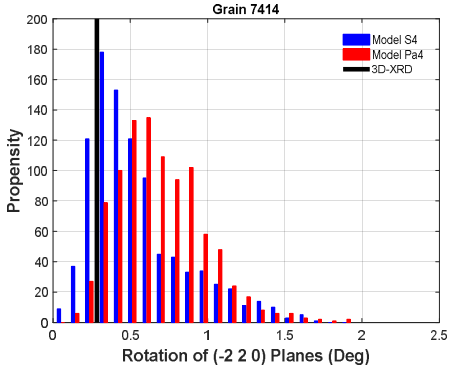

b.

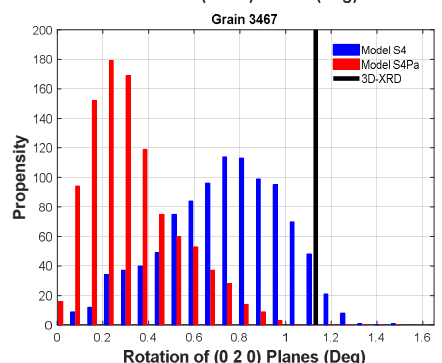

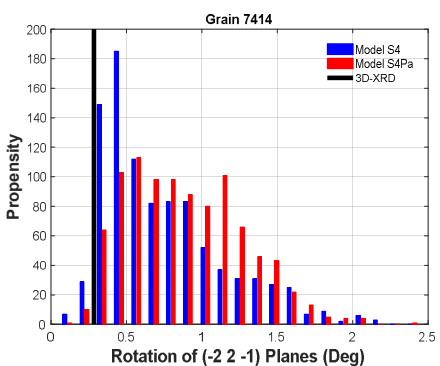

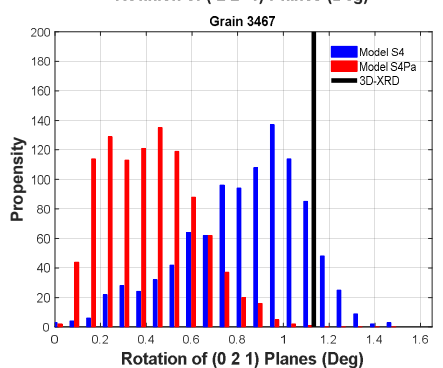

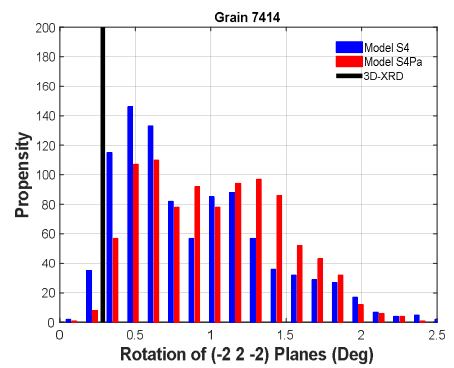

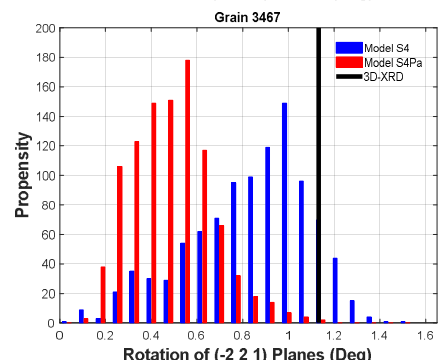

Fig. 10. Comparison between the results of the CPFE models and 3DXRD. Measured and calculated rotations for various crystallographic planes of the grains (a) 7414 and (b) 3467 . IP values are used to generate modelling results. 


\section{Supplementary File}
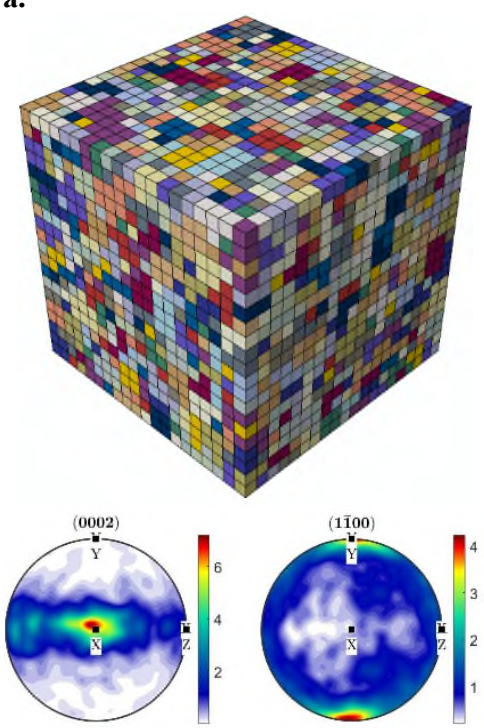

d.

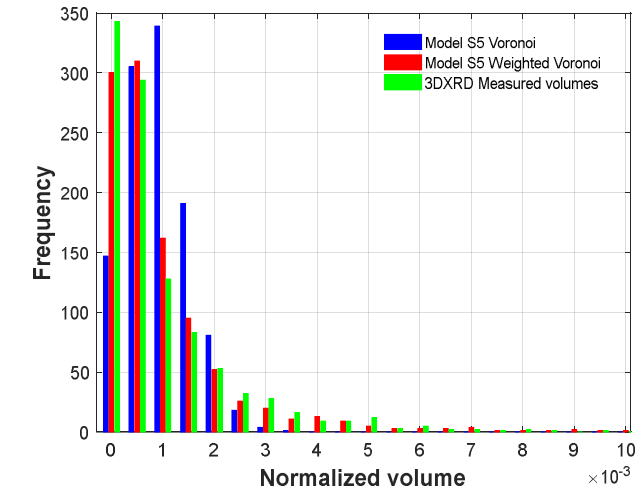

b.
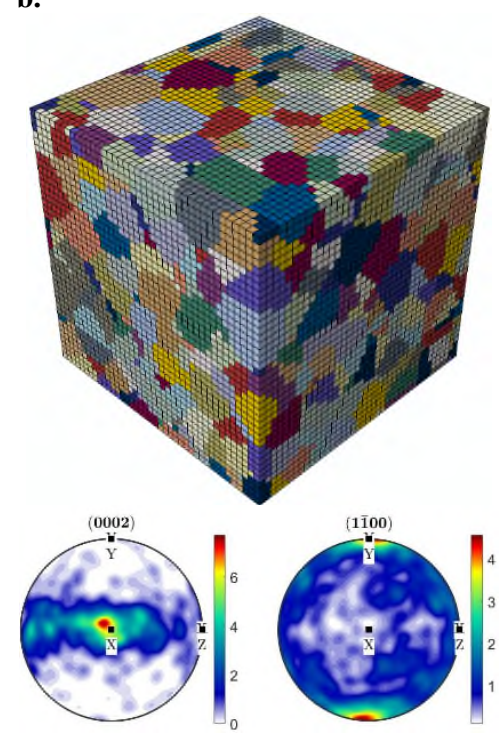

c.
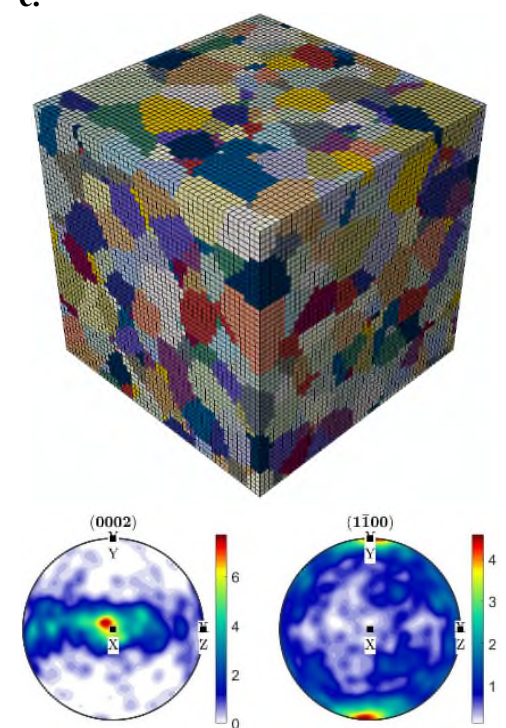

d.

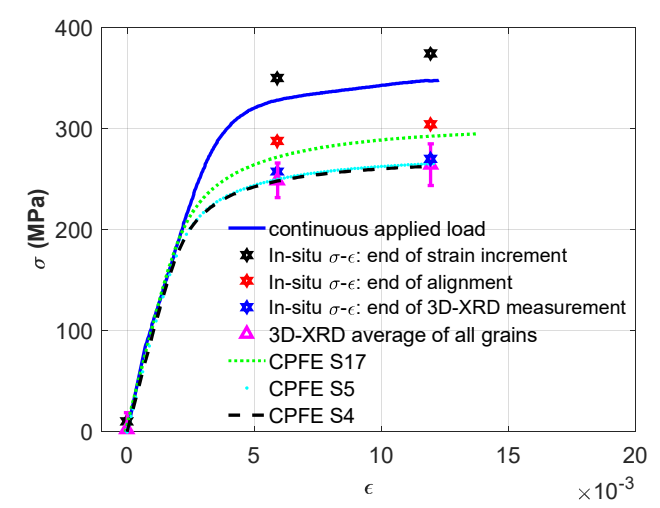

Fig. S1. Results of the modelled microstructures that were imported into a finite element solver: (a) Model S17 used for simulating 2963 grains; (b) Model S5 and (c) model S4 used for simulating 1038 grains. The pole figure that each model represents is shown below the model and random colours were assigned to elements to distinguish different grains. (d) Comparison between the measured volumes of the grains and the simulated ones using Voronoi and weighted Voronoi tessellation. (e) Comparison between the average stress-strain curves calculated for each model with the experimentally measured ones.

Model S17: A $340 \times 340 \times 340 \mu \mathrm{m}^{3}$ cube, meshed with the step size of $17 \mu \mathrm{m}$, has $20 \times 20 \times 20$ elements and covers 2963 grains.

Model S5: A $200 \times 200 \times 200 \mu \mathrm{m}^{3}$ cube, meshed with the step size of $5 \mu \mathrm{m}$, has $40 \times 40 \times 40$ elements and covers 1038 grains.

Model S4: A $200 \times 200 \times 200 \mu \mathrm{m}^{3}$ cube, meshed with the step size of $4 \mu \mathrm{m}$, has $50 \times 50 \times 50$ elements and covers 1038 grains.

In models a to c, number of integration points assigned to each grain (IP/grain) on average are 22, 493, and 963, respectively. 
a.

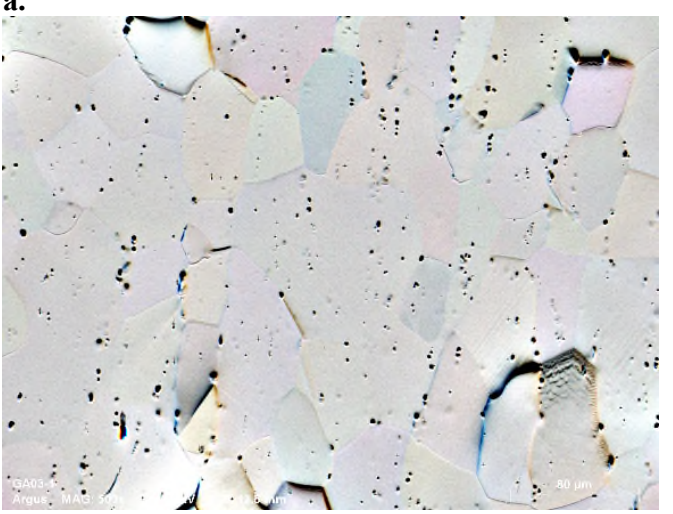

b.

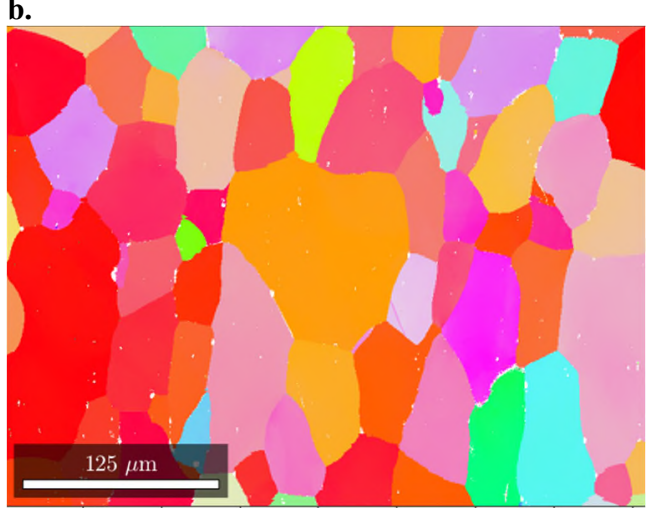

Fig. S2. (a) A forward scattering (ARGUS) image and (b) EBSD map of the deformed sample showing minimum activity of twins. 


\begin{tabular}{lcccc}
\hline & \multicolumn{3}{c}{ Supplementary Table 1 } \\
\hline & CRSS S4 & CRSS Pa4 & $n$ & $\dot{\gamma}_{0}$ \\
Prism & 82 & 95 & 20 & 0.00035 \\
Basal & 109 & 127 & 20 & 0.00035 \\
Pyramidal $<$ c + a $>$ & 287 & 287 & 20 & 0.0001 \\
Pyramidal $<a>$ & NA & 95 & 20 & 0.00035 \\
\hline
\end{tabular}

